\title{
An Approach Using Adaptive Weighted Least Squares Support Vector Machines Coupled with Modified Ant Lion Optimizer for Dam Deformation Prediction
}

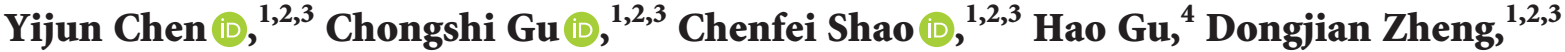 \\ Zhongru Wu iD, ${ }^{1,2,3}$ and Xiao Fu (iD) $1,2,3$ \\ ${ }^{1}$ College of Water Conservancy and Hydropower Engineering, Hohai University, Nanjing 210098, China \\ ${ }^{2}$ State Key Laboratory of Hydrology-Water Resources and Hydraulic Engineering, Hohai University, Nanjing 210098, China \\ ${ }^{3}$ National Engineering Research Center of Water Resources Efficient Utilization and Engineering Safety, Hohai University, \\ Nanjing 210098, China \\ ${ }^{4}$ Hydraulic Engineering Department, Nanjing Hydraulic Research Institute, Nanjing 210029, China
}

Correspondence should be addressed to Chongshi Gu; csgu@hhu.edu.cn

Received 20 January 2020; Accepted 13 March 2020; Published 13 April 2020

Academic Editor: Alessandro Della Corte

Copyright $\odot 2020$ Yijun Chen et al. This is an open access article distributed under the Creative Commons Attribution License, which permits unrestricted use, distribution, and reproduction in any medium, provided the original work is properly cited.

\begin{abstract}
A dam deformation prediction model based on adaptive weighted least squares support vector machines (AWLSSVM) coupled with modified Ant Lion Optimization (ALO) is proposed, which can be utilized to evaluate the operational states of concrete dams. First, the Ant Lion Optimizer, a novel metaheuristic algorithm, is used to determine the punishment factor and kernel width in the least squares support vector machine (LSSVM) model, which simulates the hunting process of antlions in nature. Second, aiming to solve the premature convergence phenomenon, Levy flight is introduced into the ALO to improve the global optimization ability. Third, according to the statistical characteristics of the datum error, an improved normal distribution weighting rule is applied to update the weighted value of data samples based on the learning result of the LSSVM model. Moreover, taking a concrete arch dam in China as an example, the horizontal displacement recorded by a pendulum is used as a study object. The accuracy and validity of the proposed model are verified and evaluated based on the four evaluating criteria, and the results of the proposed model are compared with those of well-established models. The simulation results demonstrate that the proposed model outperforms other models and effectively overcomes the influence of outliers on the performance of the model. It also has high prediction accuracy, produces excellent generalization performance, and can be a promising alternative technique for the analysis and prediction of dam deformation and other fields, including flood interval prediction, the stock price market, and wind speed forecasting.
\end{abstract}

\section{Introduction}

A dam is a significant infrastructure project that integrates flood control, hydroelectric power, irrigation, navigation, and water supply, which can facilitate the development of the social economy for a nation. China has built 97246 reservoir dams, and 756 dams are under construction, which is the largest number of reservoir dams worldwide $[1,2]$. Some dams are built in high mountain valley areas with complex topography and geological conditions and may suffer from strong earthquakes, extreme weather, and other adverse factors during the operational process. Due to the influence of large total water thrust and high dam stress, dam failure could cause large losses to the economy and to the lives of people and property. This implies that structural health monitoring is an important basis for judging the operational situation of a dam. Usually, dam deformation is the most direct reflection of the working status of a dam, and its deformation system is a typically complex dynamic system with multiple variables, strong coupling, and 
uncertainty. The analysis of the measured data and the construction of the dam deformation prediction model are determinants in structural health monitoring [3-5].

On the one hand, many random factors comprehensively affect the structural behavior of a dam, such as hydrostatic pressure, seasonal environmental temperature changes, and the aging effect [6]. Thus, dam prototype monitoring data show volatility and nonlinearity features, which increase the computational complexity of the modeling process. On the other hand, due to the interference of many uncertain factors, such as the monitoring instruments, external environment, and human factors, the noise pollution is inevitable in prototype monitoring data, which leads to a reduction in the validity of the dam deformation series, introduces difficultly in reflecting the real deformation status of the dam, and reduces the accuracy of the data analysis results [3]. Therefore, due to the nonlinearity and irregularity of the monitoring time series, an effective and reasonable prediction technique urgently needs to be introduced to ensure the safe operation of a dam based on prototype monitoring data.

To date, various powerful prediction models have been applied in complex nonlinearity and optimization problems in pump turbine characteristics identified [7], flood interval prediction [8], stock price prediction [9], and wind speed forecasting [10]. In the field of dam deformation prediction based on prototypical observations, many prediction models have also been established, such as multiple linear regression [11], neural network [5], support vector machines [12], extreme learning machine [13], boosted regression trees [14], and Gaussian process regression [15]. Nevertheless, the above-mentioned models are single prediction models, and the information mining scale is limited [16]. Meanwhile, the time series of dam deformation has high nonlinearity and outliers, which further limits the predictive power of the models. To the best of our knowledge, few prediction models take noise pollution into account in a dam deformation time series. Exploring the influence of outliers in a dam deformation time series on the prediction performance is highlighted in our work.

Support vector machines (SVM) is an important new methodology based on statistical learning theory, as introduced by Vapnik in 1995 [17], and has been used in the framework of nonlinear black-box system identification $[12,18]$. However, the SVM involves solving a quadratic programming problem and suffers from the existence of many local minima. Suykens J.A.K presented least squares support vector machines (LSSVM) by considering equality type constraints instead of inequalities from the classical SVM approach [19]. The SVM quadratic programming problem was transformed into the solution problem of the linear equation set, which had shown a better robust performance and low computational cost. However, LSSVM solutions have some potential drawbacks, including the sparseness being lost and the use of a sum squared error cost function that might obtain less robustness. Suykens applied a weighted version of the LSSVM to obtain robust estimates for regression [20], inspired by the application of a sparse robust least squares support vector machine model in the model identification of a pumped turbine governing system [21]. Meanwhile, because the dam prototype monitoring data are easily affected by outliers, the accuracy and reliability of the dam deformation prediction model are directly affected. Here, we present a novel model based on adaptive weighted least squares support vector machines (AWLSSVM) for dam deformation predictions. The proposed model inherits the advantage of the LSSVM fast learning and adaptively assigns different weight values to each training sample by using the improved normal distribution weighting rules to eliminate the influence with respect to outliers on the monitoring data and improve the anti-interference ability of the model [22].

In general, the parameters of the punishment factor and kernel width have a great influence on the prediction accuracy and generalization ability of the LSSVM model, which is generally regarded as a combinatorial optimization problem. Recently, machine learning algorithms have been widely utilized for optimizing problems and have shown superior performance. Examples of swarm intelligence algorithms include but are not limited to particle swarm optimization (PSO) [23], gravitational search algorithm (GSA) [24], ant colony optimization (ACO) [25], grey wolf optimization (GWO) [26], cuckoo search (CS) [27], grasshopper optimization approaches (GOA) [28], and the salp swarm algorithm (SSA) [29]. The Ant Lion Optimizer (ALO) algorithm is a recent swarm intelligence optimizer proposed by Mirjalili [30]. ALO simulates the preying behavior of an antlion and has a simple principle and less parameter setting. Currently, ALO is well known in identification, control theory, and signal processing [31]. However, the standard ALO has the disadvantage of early prematurity and slow convergence in the later stage. For solving this problem, different strategies have been introduced into the ALO to improve the performance [32-34]. Furthermore, Levy flight is a special random walks strategy and a reliable technique for improving group diversity $[35,36]$. This motivated our attempts to construct a modified ALO algorithm by combining Levy flights to help the ALO enhance the global search. We applied the proposed MALO algorithm to optimize the combinatorial parameters of the AWLSSVM model, which can be superior to other metaheuristic algorithms in terms of robustness, convergence speed, and avoiding local minima.

Hence, a hybrid dam deformation prediction technique based on the AWLSSVM model coupled with the MALO algorithm is constructed. The main purpose of this study is to investigate the applicability and capability of the proposed MALO-AWLSSVM model by comparing it with other models for predicting dam deformation. The remainder of this paper is structured as follows. Section 2 illustrates the choice of the influencing factors of dam deformation. Section 3 describes the construction principle of the AWLSSVM model. The parameter optimization of the AWLSSVM model using the MALO algorithm is detailed in Section 4. Section 5 displays the construction process of the dam deformation prediction model by integrating the MALO algorithm and the AWLSSVM model. In Section 6, an actual dam is taken as an example, and the experimental 
results and prediction accuracy are validated through real dam deformation time series. Finally, the conclusions are presented in Section 7.

\section{The Choice of the Influencing Factors of Dam Deformation}

According to the analysis of the structure and mechanics theory of concrete dams, the displacement of a concrete dam at any point can be divided into three components under loads of water pressure $(H)$, temperature $(T)$, and aging effect by equation (1) [6]:

$$
\delta=\delta_{H}+\delta_{T}+\delta_{\theta},
$$

where $\delta_{H}$ is the water pressure component, $\delta_{T}$ represents the temperature components, and $\delta_{\theta}$ denotes the aging effect.
2.1. Water Pressure Component. Under the action of water pressure, the horizontal displacement of a concrete dam is mainly composed of three parts in equation (2): $\delta_{1 H}$ denotes the dam displacement generated by the dam itself under the reservoir water; $\delta_{2 H}$ is the dam displacement due to the dam foundation deformation; and $\delta_{3 H}$ denotes the dam displacement because of the dam foundation rotation derived from the reservoir water gravity. Schematic diagrams are shown in Figure 1.

$$
\delta_{H}=\delta_{1 H}+\delta_{2 H}+\delta_{3 H}
$$

To simplify the calculation, the dam section is simplified as a triangular wedge that is vertical upstream, and the calculation diagram is shown in Figure 2. Under the action of reservoir water pressure, the deformation of the dam body and the foundation are generated. Thus, the theoretical formulas of $\delta_{1 H}$ and $\delta_{2 H}$ at the observation point $\mathrm{A}$ can be derived as follows:

$$
\begin{aligned}
\delta_{1 H}= & \frac{\gamma_{0}}{E_{c} m^{3}}\left[(h-d)^{2}+6(h-H)\left(d \ln \frac{h}{d}+d-h\right)+6(h-H)^{2}\left(\frac{d}{h}-1+\ln \frac{h}{d}\right)-\frac{(h-H)^{3}}{h^{2} d}(h-d)^{2}\right] \\
& +\frac{\gamma_{0}}{G_{c} m}\left[\frac{h^{2}-d^{2}}{4}-(h-H)(h-d)+\frac{(h-H)^{2}}{2} \ln \frac{h}{d}\right], \\
\delta_{2 H}= & {\left[\frac{3\left(1-\mu_{r}^{2}\right) \gamma_{0}}{\pi E_{r} m^{2} h^{2}} H^{3}+\frac{\left(1+\mu_{r}\right)\left(1-2 \mu_{r}\right) \gamma_{0}}{2 E_{r} m h_{d}} H^{2}\right](h-d), }
\end{aligned}
$$

where $H$ denotes the upstream water depth, $h$ is the height of the dam, $m$ denotes the slope of the dam downstream, $d$ is the distance between the observation point and dam crest, $E_{c}$ and $G_{c}$ are the elastic modulus and shear modulus of the dam concrete, respectively, $E_{r}$ and $\mu_{r}$ are the elastic modulus and Poisson's ratio of the dam foundation, respectively, and $\gamma_{0}$ denotes water density.

In general, the theoretical derivation for formula $\delta_{3 H}$ at the observation point A usually assumes that the reservoir bottom is level with an equal width, and the calculation diagram is shown in Figure 3. According to the solution for the uniform load applied on the surface of an infinite elastic body, the formula $\delta_{3 H}$ at observation point $\mathrm{A}$ can be derived as follows:

$$
\begin{aligned}
\delta_{3 H} & =\alpha(h-d), \\
\alpha & =\frac{\gamma_{0}\left(1+\mu_{r}\right) H}{\pi E_{r}} \ln \left(\frac{C}{x_{0}}+\sqrt{\left(\frac{C}{x_{0}}\right)^{2}+1}\right),
\end{aligned}
$$

where $\alpha$ denotes the rotation angle at the dam heel, $C$ denotes half the width of the ideal reservoir bottom, and $x_{0}$ is the distance from the centroid of the dam to the upstream dam face.

From equations (3)-(5), we can conclude that $\delta_{1 H}$ is linearly related to $H, H^{2}$, and $H^{3}$. $\delta_{2 H}$ is linearly related to
$H^{2}$ and $H^{3} . \delta_{3 H}$ is linearly related to $H$. Therefore, the water pressure component $\delta_{H}$ of the concrete gravity dam has a linear relationship with the water depth $H, H^{2}$, and $H^{3}$. For arch dams and multiarch dams, the water pressure component $\delta_{H}$ is more complicated. The arch cantilever method is usually applied to accurately analyze the stress characteristics of the arch dam. The water pressure assigned to the beam part of the arch dam is nonlinear. Similarly, the water pressure in the arch part of the multiarch dam is nonlinear due to the two-direction action of the arch part. Thus, we add $H^{4}$ as an explanatory variable for arch dams and multiarch dams. Normally, the water pressure component $\delta_{H}$ can be expressed as follows:

$$
\delta_{H}=\sum_{i=1}^{n} a_{i} H^{i},
$$

where $a_{i}$ denotes the fitting coefficient; for the concrete gravity dam, $n=3$, whereas $n=4$ is taken for the arch dam and multiarch dam.

2.2. Temperature Component. The temperature component $\delta_{T}$ is mainly composed of the dam displacement caused by the temperature variation in the dam body concrete and the dam foundation rock. The temperature is measured by embedded thermometers in the dam body and its foundation. The thermometers are usually laid out in two cases. 


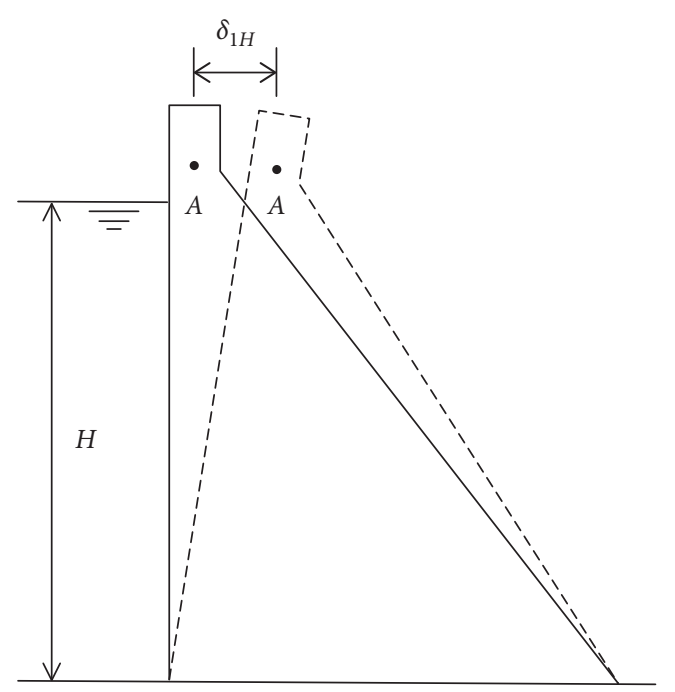

(a)

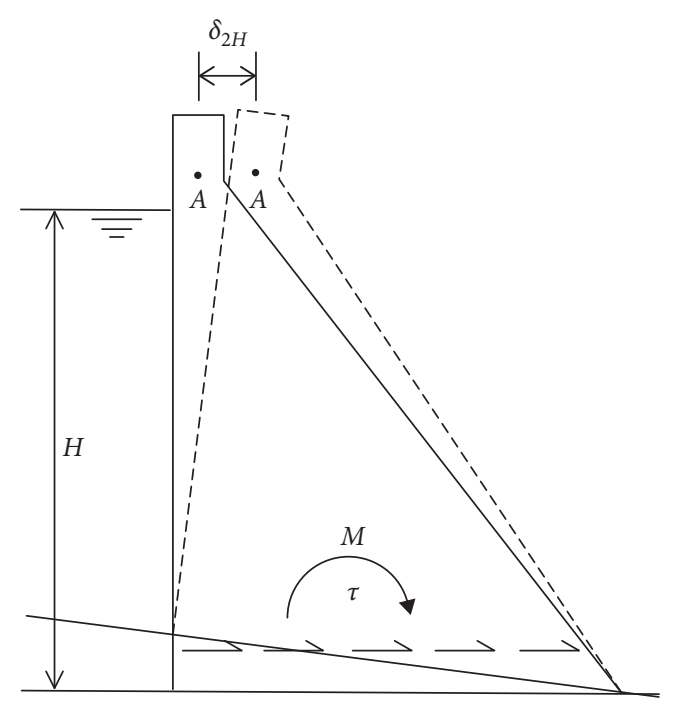

(b)

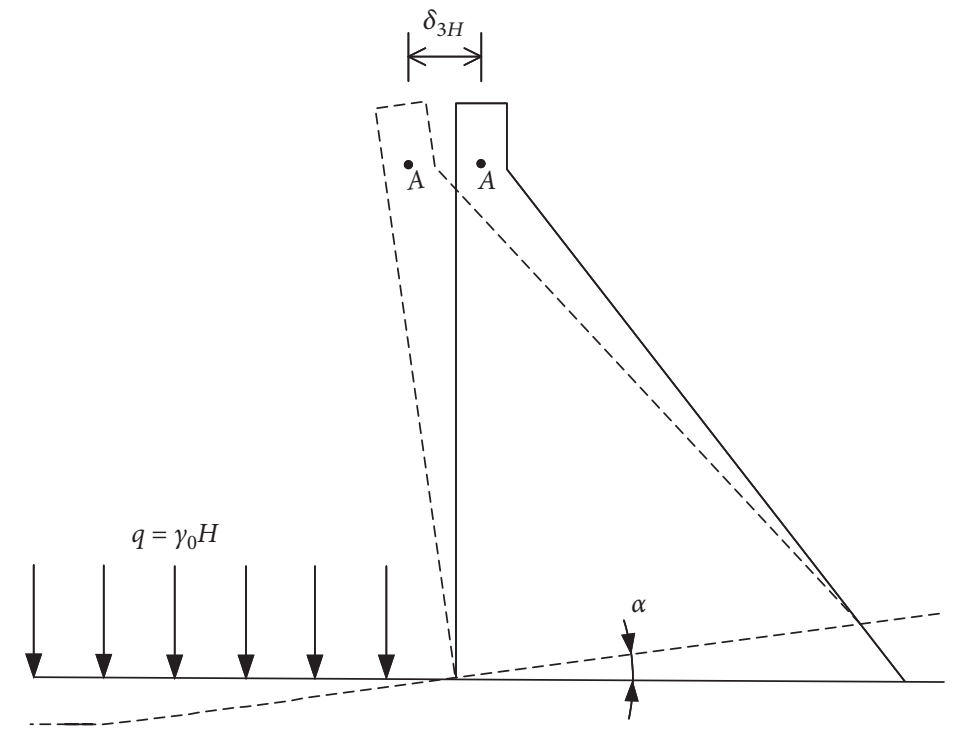

(c)

FIgURE 1: Schematic diagram: (a) $\delta_{1 H}$, (b) $\delta_{2 H}$, and (c) $\delta_{3 H}$.

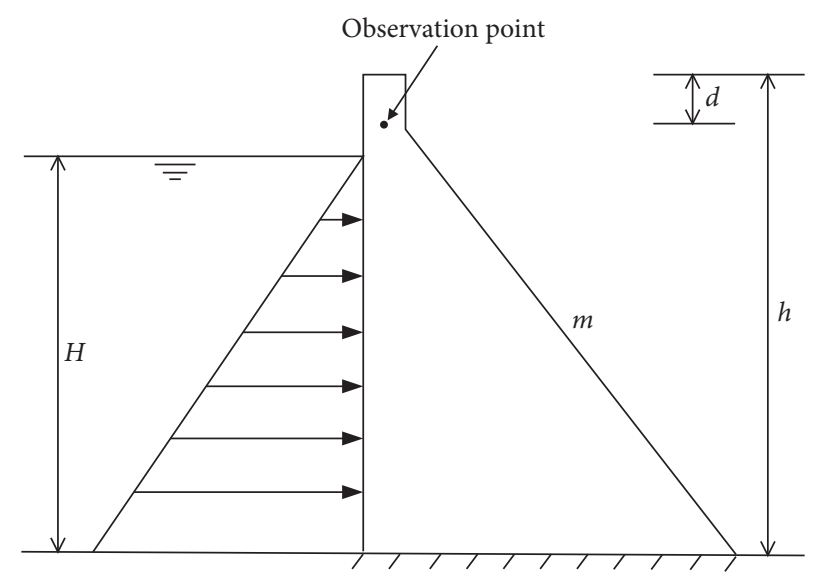

Figure 2: Calculation diagram of $\delta_{1 H}$ and $\delta_{2 H}$. 


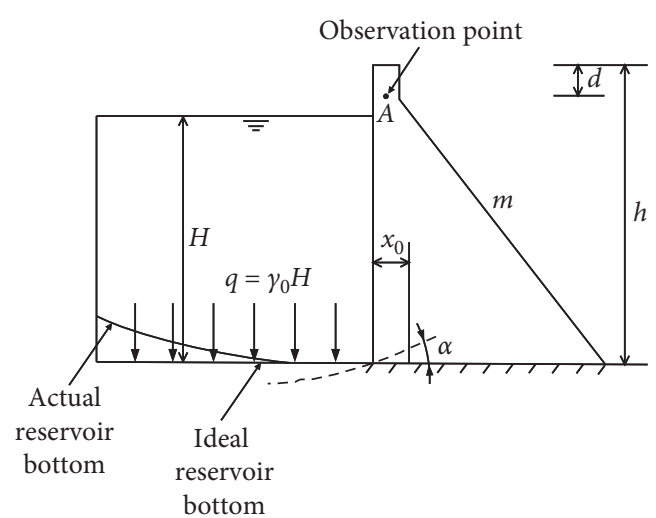

(a)

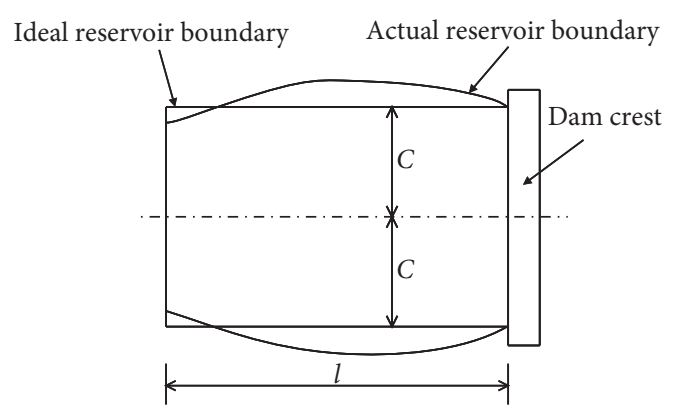

(b)

FIgURE 3: Calculation diagram of $\delta_{3 H}$ : (a) side view; (b) vertical view.

In the first case, there are adequate monitoring data from the thermometers reflecting the temperature field, and the temperature component $\delta_{T}$ can be given by equations (8) and (9):

$$
\begin{aligned}
& \delta_{T}=\sum_{i=1}^{m_{1}} b_{i} T_{i}, \\
& \delta_{T}=\sum_{i=1}^{m_{2}} b_{1 i} \bar{T}_{i}+\sum_{i=1}^{m_{2}} b_{2 i} \beta_{i},
\end{aligned}
$$

where $b_{i}, b_{1 i}$, and $b_{2 i}$ are the fitting coefficients, $T_{i}$ represents the thermometer temperature value, $m_{1}$ denotes the number of thermometers, $\bar{T}_{i}$ and $\beta_{i}$ represent the mean value and gradient of variation of the equivalent temperature in the $i$ th layer, respectively, and $m_{2}$ denotes the number of layers with thermometers installed.

In the second case, the temperature of the dam body concrete and dam foundation rock are not monitored by thermometers. It is assumed that the hydration heat of the concrete has been distributed and the temperature in the dam body reaches a quasi-steady temperature field; then the multiperiod harmonic can be selected to represent the temperature component as

$$
\delta_{T}=\sum_{i=1}^{m}\left(b_{1 i} \sin \frac{2 \pi i t}{365}+b_{2 i} \cos \frac{2 \pi i t}{365}\right),
$$

where $i$ denotes the period, for the annual cycle $m=1$, for a half cycle $m=2, b_{1 i}$ and $b_{2 i}$ represent the fitting coefficients, and $t$ is the cumulative number of days from the initial value to the monitoring value.

2.3. Aging Component. The causes of the aging component of the dam are complex. The aging component reflects the creep and plastic deformation of the dam concrete and the foundation rock, as well as the compression deformation of the foundation rock geological structure. It also includes the autogenous volume deformation and the irreversible displacement caused by dam cracks. The mathematical model of the aging component can be written by equation (11), where $\theta=(t / 100)$ :

$$
\delta_{\theta}=c_{1} \theta+c_{2} \ln \theta \text {, }
$$

where $c_{1}$ and $c_{2}$ are the fitting coefficients for the aging factors.

2.4. Statistical Model Expression. In summary, according to the concrete dam characteristics, three kinds of statistical models for concrete dam horizontal displacement are depicted in equations (12)-(14). In the application, the most appropriate statistical model is selected according to the concrete project example.

$$
\begin{aligned}
& \delta=a_{0}+\sum_{i=1}^{n} a_{i} H^{i}+\sum_{i=1}^{m} b_{i} T_{i}+c_{1} \theta+c_{2} \ln \theta, \\
& \delta=a_{0}+\sum_{i=1}^{n} a_{i} H^{i}+\sum_{i=1}^{m_{2}} b_{1 i} \bar{T}_{i}+\sum_{i=1}^{m_{2}} b_{2 i} \beta_{i}+c_{1} \theta+c_{2} \ln \theta, \\
& \delta=a_{0}+\sum_{i=1}^{n} a_{i} H^{i}+\sum_{i=1}^{m}\left(b_{1 i} \sin \frac{2 \pi i t}{365}+b_{2 i} \cos \frac{2 \pi i t}{365}\right)+c_{1} \theta+c_{2} \ln \theta,
\end{aligned}
$$

where $a_{0}$ denotes the constant term considering the influence of the initial state and the other symbols have the same meaning as above.

\section{The Construction Principle of the AWLSSVM Model for Predicting Dam Deformation}

3.1. Least Squares Support Vector Machine Regression. The LSSVM is a modification of the standard SVM model. The formulation consists of equality constraints instead of inequality constraints and a sum squared error cost function as it is frequently used in the process of training. Hence, the complexity and difficulty associated with modeling are greatly reduced, and the solving speed is effectively improved. The LSSVM is used for the optimal control of 
nonlinear Karush-Kuhn-Tucker systems for classification and regression [19].

Suppose a training data set is $G=\left\{\left(\mathbf{x}_{1}, y_{1}\right)\right.$, $\left.\left(\mathbf{x}_{2}, y_{2}\right), \ldots,\left(\mathbf{x}_{N}, y_{N}\right)\right\}(N$ is the number of training sample pairs), where $\mathbf{x}_{k} \in R^{n}(k=1,2, \ldots, N)$ denotes the space of the input patterns and $y_{k} \in R$ represents the space of the output patterns. The LSSVM model can be expressed as

$$
y(\mathbf{x})=\boldsymbol{\omega}^{T} \varphi(\mathbf{x})+b .
$$

The optimization problem of the LSSVM model can be described as

$$
\begin{cases}\min & J\left(\boldsymbol{\omega}, \xi_{i}\right)=\frac{1}{2} \boldsymbol{\omega}^{T} \boldsymbol{\omega}+\frac{1}{2} \gamma \sum_{i=1}^{N} \xi_{i}^{2} \\ \text { s.t. } & y_{i}=\boldsymbol{\omega}^{T} \varphi\left(\mathbf{x}_{i}\right)+b+\xi_{i}, \quad i=1,2, \ldots, N,\end{cases}
$$

where $\boldsymbol{\omega} \in R^{n_{h}}$ denotes the weight coefficient vector, $\gamma$ represents the punishment factor, which determines the tradeoff between the complexity of the LSSVM model and its accuracy in capturing the training data, $b$ denotes the bias term, $\xi_{i} \in R$ is the fitting error, and $\varphi(\cdot): R^{n} \longrightarrow R^{n_{h}}$ is a function that maps the input space into a so-called higherdimensional (possibly infinite-dimensional) feature space. For the optimization problem, the Lagrange function is constructed as

$$
L(\boldsymbol{\omega}, b, \boldsymbol{\xi}, \boldsymbol{\alpha})=\frac{1}{2} \boldsymbol{\omega}^{T} \boldsymbol{\omega}+\frac{1}{2} \gamma \sum_{i=1}^{N} \xi_{i}^{2}-\sum_{i=1}^{N} \alpha_{i}\left(\boldsymbol{\omega}^{T} \boldsymbol{\varphi}\left(\mathbf{x}_{i}\right)+b+\xi_{i}-y_{i}\right),
$$

where $\alpha_{i} \in R(i=1,2, \ldots, N)$ denotes the introduced Lagrange multiplier. According to the Karush-Kuhn-Tucker optimization conditions, equation (18) must be satisfied:

$$
\left\{\begin{array}{l}
\frac{\partial L}{\partial \boldsymbol{\omega}}=0 \longrightarrow \boldsymbol{\omega}=\sum_{i=1}^{N} \alpha_{i} \boldsymbol{\varphi}\left(\mathbf{x}_{i}\right), \\
\frac{\partial L}{\partial b}=0 \longrightarrow \sum_{i=1}^{N} \alpha_{i}=0, \\
\frac{\partial L}{\partial \xi_{i}}=0 \longrightarrow \alpha_{i}=\gamma \xi_{i}, \quad i=1,2, \ldots, N, \\
\frac{\partial L}{\partial a_{i}}=0 \longrightarrow \boldsymbol{\omega}^{T} \boldsymbol{\varphi}\left(\mathbf{x}_{i}\right)+b+\xi_{i}-y_{i}=0, \quad i=1,2, \ldots, N .
\end{array}\right.
$$

After eliminating the variables $\boldsymbol{\xi}$ and $\boldsymbol{\omega}$, the optimization problem of equation (16) can be changed into the solution problem of the following matrix:

$$
\left[\begin{array}{cc}
0 & \mathbf{1}_{1 \times N} \\
\mathbf{l}_{N \times 1} & \mathbf{R}+\frac{1}{\gamma}
\end{array}\right]\left[\begin{array}{l}
b \\
\boldsymbol{a}
\end{array}\right]=\left[\begin{array}{l}
0 \\
\mathbf{y}
\end{array}\right],
$$

where $\quad \mathbf{l}_{1 \times N}=[1,1, \ldots, 1], \quad \mathbf{l}_{N \times 1}=[1,1, \ldots, 1]^{T}$, $\mathbf{y}=\left[y_{1}, y_{2}, \ldots, y_{N}\right]^{T}, \boldsymbol{\alpha}=\left[\alpha_{1}, \alpha_{2}, \ldots, \alpha_{N}\right]^{T}$, and $K\left(\mathbf{x}_{i}, \mathbf{x}_{j}\right)=$ $\boldsymbol{\varphi}^{T}\left(\mathbf{x}_{i}\right) \boldsymbol{\varphi}\left(\mathbf{x}_{j}\right)$ is a kernel function that represents the highdimensional feature spaces. There are four commonly used kernel functions in Table 1 [37]. $\mathbf{R}$ can be expressed as

$$
\mathbf{R}=\left[\begin{array}{cccc}
K\left(\mathbf{x}_{1}, \mathbf{x}_{1}\right) & K\left(\mathbf{x}_{1}, \mathbf{x}_{2}\right) & & K\left(\mathbf{x}_{1}, \mathbf{x}_{N}\right) \\
K\left(\mathbf{x}_{2}, \mathbf{x}_{1}\right) & K\left(\mathbf{x}_{2}, \mathbf{x}_{2}\right) & & K\left(\mathbf{x}_{2}, \mathbf{x}_{N}\right) \\
\vdots & \ddots & \vdots \\
K\left(\mathbf{x}_{N}, \mathbf{x}_{1}\right) & K\left(\mathbf{x}_{N}, \mathbf{x}_{2}\right) & \cdots & K\left(\mathbf{x}_{N}, \mathbf{x}_{N}\right)
\end{array}\right] .
$$

The parameters $\boldsymbol{\alpha}$ and $b$ can be solved with equation (19), and the resulting LSSVM model for function estimation is given by

$$
y=\sum_{i=1}^{N} \alpha_{i} K\left(\mathbf{x}_{i}, \mathbf{x}\right)+b
$$

3.2. Weighted Least Squares Support Vector Machine Regression. Suykens et al. proposed a weighted LSSVM model based on the standard LSSVM model, which can eliminate the influence of outliers in the training sample on the predictive performance and guarantee good generalization performance [20-22]. The optimization problem can be rewritten as

$$
\begin{cases}\min & J^{*}\left(\boldsymbol{\omega}, \xi_{i}\right)=\frac{1}{2} \boldsymbol{\omega}^{T} \boldsymbol{\omega}+\frac{1}{2} \gamma \sum_{i=1}^{N} v_{i} \xi_{i}^{2} \\ \text { s.t. } & y_{i}=\boldsymbol{\omega}^{T} \varphi\left(\mathbf{x}_{i}\right)+b+\xi_{i}, \quad i=1,2, \ldots, N,\end{cases}
$$

where $v_{i}$ denotes the weight value and the other symbols have the same meaning as above.

The Lagrange function becomes

$$
L\left(\boldsymbol{\omega}, b, \xi, \boldsymbol{\alpha}^{*}\right)=\frac{1}{2} \boldsymbol{\omega}^{T} \boldsymbol{\omega}+\frac{1}{2} \gamma \sum_{i=1}^{N} v_{i} \xi_{i}^{2}-\sum_{i=1}^{N} \alpha_{i}^{*}\left(\boldsymbol{\omega}^{T} \boldsymbol{\varphi}\left(\mathbf{x}_{i}\right)+b+\xi_{i}-y_{i}\right),
$$

where $\alpha_{i}^{*}(i=1,2, \ldots, N)$ denotes the introduced Lagrange multiplier. According to the Karush-Kuhn-Tucker systems, after optimizing equation (22) and eliminating $\boldsymbol{\xi}$ and $\boldsymbol{\omega}$, the solution can be given by

$$
\left[\begin{array}{cc}
0 & \mathbf{1}_{1 \times N} \\
\mathbf{l}_{N \times 1} & \mathbf{R}+\frac{\mathbf{V}}{\gamma}
\end{array}\right]\left[\begin{array}{l}
b \\
\mathbf{a}^{*}
\end{array}\right]=\left[\begin{array}{l}
0 \\
\mathbf{y}
\end{array}\right],
$$

where the diagonal matrix is $\mathbf{V}=\operatorname{diag}\left(v_{1}^{-1}, v_{2}^{-1}, \ldots, v_{N}^{-1}\right)$, $v_{i}(i=1,2, \ldots, N)$ denotes the weight variable, and the other parameters are the same as before. Finally, the nonlinear system function can be described as

$$
y=\sum_{i=1}^{N} \alpha_{i}^{*} K\left(\mathbf{x}_{i}, \mathbf{x}\right)+b .
$$


TABLE 1: Different types of kernel functions.

\begin{tabular}{lc}
\hline Name of kernel & Expression \\
\hline Linear kernel & $K\left(\mathbf{x}_{i}, \mathbf{x}_{j}\right)=\mathbf{x}_{i} \cdot \mathbf{x}_{j}+\theta$ \\
Polynomial kernel & $K\left(\mathbf{x}_{i}, \mathbf{x}_{j}\right)=\left(\mathbf{x}_{i} \mathbf{x}_{j}+1\right)^{p}$ \\
Gaussian radial basis & $K\left(\mathbf{x}_{i}, \mathbf{x}_{j}\right)=\exp \left\{-\left\|\mathbf{x}_{i}-\mathbf{x}_{j}\right\|_{2}^{2} /\left(2 \sigma^{2}\right)\right\}$ \\
function (RBF) & $K\left(\mathbf{x}_{i}, \mathbf{x}_{j}\right)=\tanh \left\{\left(\mathbf{x}_{i} \cdot \mathbf{x}_{j}\right)+b\right\}$ \\
Sigmoid kernel &
\end{tabular}

3.3. Construction of the Adaptive Weighted Technique. To eliminate the influence of the inevitable outliers in the training set on the dam deformation prediction performance and improve the robustness of the model, the adaptive weighted technique is proposed and introduced into the standard LSSVM model [20]. The weight value $v_{i}$ can be defined as follows:

$$
v_{i}=\exp \left(\frac{-\left(\left|\xi_{i}\right|-\mu\right)^{2}}{u s}\right)
$$

where $\xi_{i}$ represents the $i$-th sample residual and $u$ denotes the adjustment coefficient, which is optimized by the MALO algorithm in this work. $\mu$ and $s$ are the mean value and standard deviation of the error absolute value $\left|\xi_{i}\right|$, respectively, and the values can be computed by

$$
\begin{aligned}
\mu & =\frac{1}{N} \sum_{i=1}^{N}\left|\xi_{i}\right|, \\
s & =\sqrt{\frac{1}{N} \sum_{i=1}^{N}\left(\left|\xi_{i}\right|-\mu\right)^{2} .}
\end{aligned}
$$

\section{Parameter Optimization of the AWLSSVM Model Using the MALO Algorithm}

4.1. The Mathematical Model of the ALO Algorithm. In this work, the RBF in equation (28) is employed as a kernel function in the LSSVM model. The RBF can construct the optimal classification hyperplane and map the input into the high-dimensional feature space to eliminate dimensional disasters. In this way, the complex nonlinear relationship between the sample input and output can be handled well [37].

$$
K\left(\mathbf{x}_{i}, \mathbf{x}_{j}\right)=\exp \left(-\frac{\left\|\mathbf{x}_{i}-\mathbf{x}_{j}\right\|_{2}^{2}}{2 \sigma^{2}}\right)
$$

where $\sigma$ denotes the kernel width.

The main parameters of the LSSVM model with the RBF are the punishment factor $\gamma$ and kernel width $\sigma$, which determine the prediction accuracy and robust performance of the LSSVM model. The punishment factor $\gamma$ determines the degree of punishment for the sample data. If the value is too large or too small, the generalization performance of the training model will become worse. The kernel width $\sigma$ controls the complexity of the final solution, and a value that is too large or too small can result in underlearning or overlearning. In the existing literature, the parameter optimization is mostly determined by trial calculation and empirical methods, which is not only time-consuming but also inaccurate $[38,39]$. In this work, the MALO algorithm is applied to select the optimal parameter combination $\left(\gamma_{\text {best }}, \sigma_{\text {best }}\right)$ to reduce the blindness of parameter selection and improve the prediction performance of the LSSVM model.

In 2015, the Ant Lion Optimizer was proposed by Mirjalili [30] as a novel nature-inspired metaheuristic algorithm that mimics the hunting mechanism of antlions in nature. It has the advantages of high optimization efficiency and high solution precision due to the use of random walks and roulette wheels. The ALO consists of exploration by random walks of ants and random selection of antlions, and exploitation of the search space is guaranteed by the adaptive shrinking boundaries of antlion traps. The ALO with an excellent search function motivates us to employ the parameter optimization algorithm in the AWLSSVM model, which has not been used earlier to the knowledge of the authors. The mathematical model of the ALO is described in the following steps.

4.1.1. Random Walks of Ants. Ants move stochastically in nature when they search for food, and the random walks of ants can be described as

$$
X^{t}=\left[0, \operatorname{cumsum}\left(2 r\left(t_{1}\right)-1\right), \operatorname{cumsum}\left(2 r\left(t_{2}\right)-1\right), \ldots, \operatorname{cumsum}\left(2 r\left(t_{\text {iter }_{\max }}\right)-1\right)\right]
$$

where $X^{t}$ denotes the random walks of ants, iter $\max$ represents the maximum number of iterations, $t$ is the step of the random walks, cumsum denotes the calculating cumulative sum, and $r(t)$ is a stochastic function defined as

$$
r\left(t_{k}\right)= \begin{cases}1, & \text { if } \text { rand }>0.5 \\ 1, & \text { if } \text { rand } \leq 0.5\end{cases}
$$

where rand denotes a random number generated with a uniform distribution in the interval of $(0,1)$.
To keep the random walks of ants inside the search space, the positions need to be normalized using the following minmax normalization equation:

$$
X_{i}^{t}=\frac{\left(X_{i}^{t}-a_{i}\right) \times\left(U_{i}^{t}-L_{i}^{t}\right)}{\left(b_{i}-a_{i}\right)}+L_{i}^{t},
$$

where $a_{i}$ and $b_{i}$ are the minimum and maximum of the random walks of the $i$-th variable, respectively, and $L_{i}^{t}$ and $U_{i}^{t}$ denote the minimum and maximum of the $i$-th variable at the $t$-th iteration, respectively. 


$$
\begin{aligned}
\mathbf{M}_{\text {Ant }} & =\left[\begin{array}{cccc}
x_{1,1} & x_{1,2} & & x_{1, d} \\
x_{2,1} & x_{2,2} & & x_{2, d} \\
\vdots & \ddots & \vdots \\
x_{N, 1} & x_{N, 2} & \cdots & x_{N, d}
\end{array}\right], \\
\mathbf{M}_{\text {Antlion }} & =\left[\begin{array}{cccc}
x_{1,1} & x_{1,2} & \ldots & x_{1, d} \\
x_{2,1} & x_{2,2} & & x_{2, d} \\
\vdots & \ddots & \vdots \\
x_{M, 1} & x_{M, 2} & \cdots & x_{M, d}
\end{array}\right],
\end{aligned}
$$

where $\mathbf{M}_{\text {Ant }}$ and $\mathbf{M}_{\text {Antlion }}$ denote the position matrix of ants and antlions, respectively, and $N$ and $M$ represent the number of ants and antlions, respectively.

To balance the model generalization and computational complexity, the following objective function is used to evaluate all ants and antlions. In general, the well-fitting of the training set can increase the complexity of the prediction model, and the complex model tends to suffer from overfitting [40].

$$
\begin{aligned}
f\left(x_{i, 1}, x_{i, 2}, \ldots, x_{i, d}\right)= & \sqrt{\frac{1}{N_{\text {train }}} \sum_{i=1}^{N_{\text {train }}}\left(y_{i}-y_{i}^{*}\right)^{2}} \\
& +\sqrt{\frac{1}{N_{\text {test }}} \sum_{i=1}^{N_{\text {test }}}\left(y_{i}-y_{i}^{*}\right)^{2}},
\end{aligned}
$$

where $f$ is the objective function, $N_{\text {train }}$ and $N_{\text {test }}$ are the numbers of samples in the training set and test set, respectively, and $y_{i}$ and $y_{i}^{*}$ are the $i$-th measured value and predicted value, respectively. The matrices $\mathbf{M}_{\mathrm{OA}}$ and $\mathbf{M}_{\mathrm{OAL}}$ are used to store the fitness values of the ants and antlions in equations (34) and (35). In this work, the antlion with a minimum fitness value will be defined as the elite antlion.

$$
\begin{gathered}
\mathbf{M}_{\mathrm{OA}}=\left[\begin{array}{c}
f\left(x_{1,1}, x_{1,2}, \ldots, x_{1, d}\right) \\
f\left(x_{2,1}, x_{2,2}, \ldots, x_{2, d}\right) \\
\vdots \\
f\left(x_{N, 1}, x_{N, 2}, \ldots, x_{N, d}\right)
\end{array}\right], \\
\mathbf{M}_{\mathrm{OAL}}=\left[\begin{array}{c}
f\left(x_{1,1}, x_{1,2}, \ldots, x_{1, d}\right) \\
f\left(x_{2,1}, x_{2,2}, \ldots, x_{2, d}\right) \\
\vdots \\
f\left(x_{M, 1}, x_{M, 2}, \ldots, x_{M, d}\right)
\end{array}\right] .
\end{gathered}
$$

4.1.2. Building Traps. To mimic the antlion's hunting capability, a roulette wheel operator is employed to select antlions based on their fitness value during the process of optimization. This mechanism produces a high probability that the fitter antlions will catch ants.
4.1.3. Trapping in Antlion Pits. As discussed above, random walks of ants are affected by antlions traps. The mathematical models for trapping are given as follows:

$$
\begin{gathered}
U_{i}^{t}=\text { Antlion }_{j}^{t}+U^{t}, \\
L_{i}^{t}=\text { Antlion }_{j}^{t}+L^{t},
\end{gathered}
$$

where Antlion ${ }_{j}^{t}$ denotes the position of the selected $j$-th antlion at the $t$-th iteration and $L^{t}$ and $U^{t}$ are the minimum and maximum of all the variables at the $t$-th iteration, respectively.

4.1.4. Sliding Ants towards Antlions. Antlions can build traps that are proportional to their fitness values during the iteration process. Antlions shoot sand outwards the center of the pits once they realize that an ant is trapped. To mathematically simulate the movement behavior of ants sliding towards antlions, the random walks range is set to decrease adaptively, which can be described as

$$
\begin{gathered}
U^{t}=\frac{U^{t}}{I}, \\
L^{t}=\frac{L^{t}}{I},
\end{gathered}
$$

where $I=10^{w}(t / T)$ is a decreased ratio, $t$ denotes the current iteration, $T$ represents the maximum number of iterations, and $w$ denotes a constant that is defined based on the current iteration $(w=2$, when $t>0.1 T ; w=3$, when $t>0.5 T ; w=4$, when $t>0.75 T ; w=5$, when $t>0.9 T$; and $w=6$, when $t>0.95 T)$.

4.1.5. Elitism. According to the elite strategy, the ant position is affected by both the antlion and the selected elite. The mathematical model of the $i$-th ant position at the $t$-th iteration can be performed by

$$
\operatorname{Ant}_{i}^{t}=\frac{R_{A}^{t}+R_{E}^{t}}{2}
$$

where $R_{A}^{t}$ and $R_{E}^{t}$ denote the random walks around the antlion selected by the roulette wheel and the selected elite, respectively.

4.1.6. Catching Prey and Rebuilding the Pit. The antlion will catch an ant when the ant reaches the pit bottom, and then it will pull the ant into the sand and consume it. To improve the probability of catching the next ant, the antlion will update its position based on the position of the latest caught ant and then build a new pit. In this work, when the fitness value of an ant is less than the antlion, the ant will be caught, and the position of the antlion is updated by

$$
\text { Antlion }_{j}^{t}=\operatorname{Ant}_{i}^{t} \text { if } f\left(\text { Ant }_{i}^{t}\right)<f\left(\text { Antlion }_{j}^{t}\right) \text {. }
$$

4.2. Levy Flight Mutation Mechanism for Ants. The ALO has problems related to premature convergence and not ideal random walks. In this work, Levy flight is utilized to perturb 
the ants to improve the mutation mechanism. Levy flight is a special random walk strategy that performs global searches more effectively and can effectively describe the foraging patterns of many biological groups [36]. As shown in Figure 4, Levy flight has a larger exploration scope and stronger development ability, while the random walks exploration scope is small and evenly dispersed. Therefore, the diversity of the whole population can be significantly improved, and the ability of local development and global exploration of the complex optimization algorithm can be effectively balanced [41].

Therefore, the Levy flight is utilized to replace the random walks for modeling the motion of ants, and equation (29) can be redefined as

$$
\begin{aligned}
X^{t}= & {\left[0, \operatorname{cumsum}\left(L\left(t_{1}\right)-1\right), \operatorname{cumsum}\left(L\left(t_{2}\right)-1\right), \ldots,\right.} \\
& \left.\operatorname{cumsum}\left(L\left(t_{\text {iter }_{\text {max }}}\right)-1\right)\right],
\end{aligned}
$$

where $L(t)$ is the step size of the random search at the $t$-th iteration and can be defined as

$$
L(t ; s, \lambda)=\frac{\lambda \Gamma(\lambda) \sin (\pi \lambda / 2)}{\pi s^{1+\lambda}},
$$

where $\Gamma$ denotes the standard gamma function. $s$ is a pseudorandom step size generated by using the Levy flight, which is a nontrivial scheme. Equation (42) is the most effective and direct method for adopting the Mantegna algorithm:

$$
s=\frac{U}{|V|^{1 / \lambda}}, \quad 1<\lambda \leq 3
$$

where $U$ and $V$ are drawn from Gaussian distributions and expressed as

$$
\begin{aligned}
& U \sim N\left(0, \sigma^{2}\right), \\
& V \sim N(0,1),
\end{aligned}
$$

where

$$
\sigma^{2}=\left[\frac{\Gamma(1+\lambda) \sin (\pi \lambda / 2)}{\lambda \Gamma((1+\lambda) / 2) 2^{(\lambda-1) / 2}}\right]^{1 / \lambda}
$$

4.3. The Construction Process of the MALO Algorithm. To summarize, a novel modified ALO is constructed in this paper by combining the Levy flights mutation mechanism for optimizing the punishment factor $\lambda$ and kernel width $\sigma$ in the LSSVM model. The pseudocode of the MALO is given in Algorithm 1.

\section{The Construction Process of the Dam Deformation Prediction Model by Integrating the MALO and AWLSSVM}

According to Sections 2-4, the dam deformation prediction model integrates the AWLSSVM model and the MALO algorithm to predict future dam deformation. The model

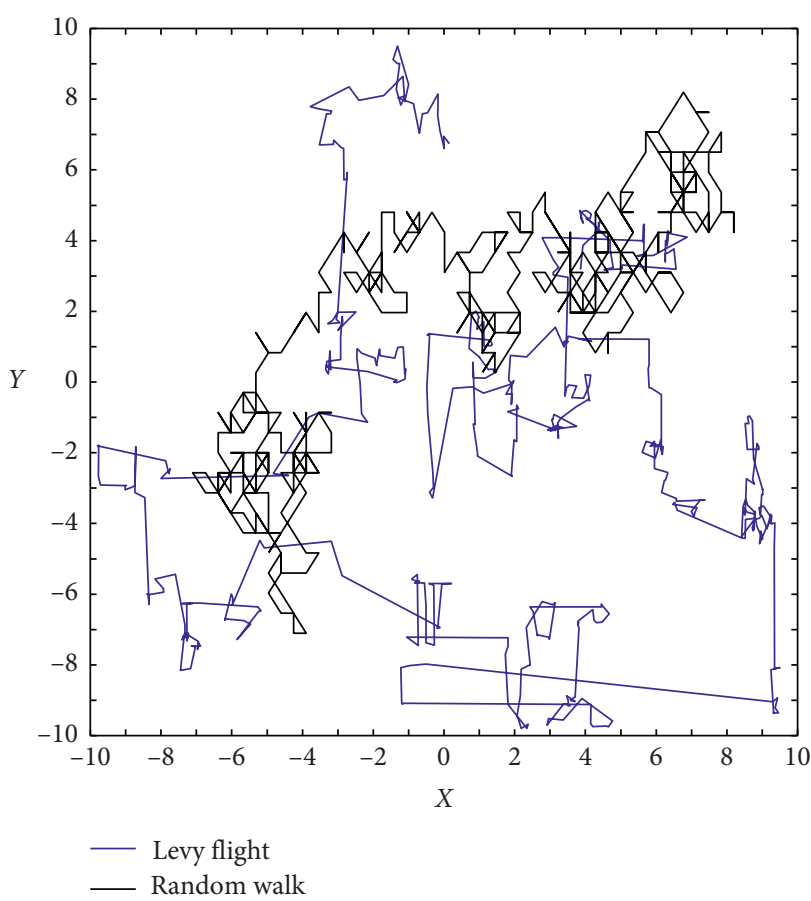

FIGURE 4: The 2D trajectory of Levy flights and random walks in the set region.

structure and operating process are shown in Figure 5 and have been implemented in the MATLAB 2014a platform. The specific operation process for dam deformation prediction is described as follows.

Step 1. Construct the data sets for the input and output patterns of the prediction model and divide the data set into a training set and a test set based on a certain proportion. As described in Section 2, the influencing factors of dam deformation, such as the water pressure component $H$, temperature component $T$, and aging component, are taken as the input variables and the dam deformation as the output pattern of the model.

According to equation (7), for the concrete arch dam, $n$ is usually taken as 4 . Therefore, the water level factors are selected as follows:

$$
\left\{H, H^{2}, H^{3}, H^{4}\right\} \text {. }
$$

Considering the lack of thermometer monitoring data and semiannual harmonics, the temperature factors are described as follows based on equation (10).

$$
\left\{\sin \frac{2 \pi t}{365}, \cos \frac{2 \pi t}{365}, \sin \frac{4 \pi t}{365}, \cos \frac{4 \pi t}{365}\right\} \text {. }
$$

According to equation (11), the aging factors are selected as follows:

$$
\{\theta, \ln \theta\} .
$$

Therefore, the input and output patterns of model can 
(1) Construct the training set and test set, initialize the parameters $\left(t_{\max }, N, M, \gamma_{\min }, \gamma_{\max }, \sigma_{\min }, \sigma_{\max }, t=0\right)$;

(2) Initialize the first population of ants and antlions by the random sampling;

(3) Construct the LSSVM model by equation (21), evaluate the fitness values of the ants and antlions by equation (34);

(4) Determine the antlion with the minimum fitness value as the elite antlion;

(5) While $t<t_{\max }$ do

(6) $\quad t=t+1$;

(7) For each ant

(8) Select an antlion based on the fitness value using the Roulette wheel principle;

(9) Update the lower and upper bounds by equation (38);

(10) Calculate the bounds around the selected antlion by equation (37);

(11) Determine $R_{A}^{t}$ and $R_{E}^{t}$, the Levy flights around the selected antlion by equations (31) and (41);

(12) Update the new state of the ants using equation (39);

(13) End for

(14) Construct the LSSVM model and evaluate the fitness values of all the ants;

(15) Update all the position of the antlions using equation (40);

(16) Update the elite antlion if an antlion becomes fitter than the elite;

(17) End while

(18) Return elite antlion, namely, the optimal parameter combination $\left(\gamma_{\text {best }}, \sigma_{\text {best }}\right)$.

Algorithm 1: Parameter optimization for the LSSVM model using the MALO algorithm.

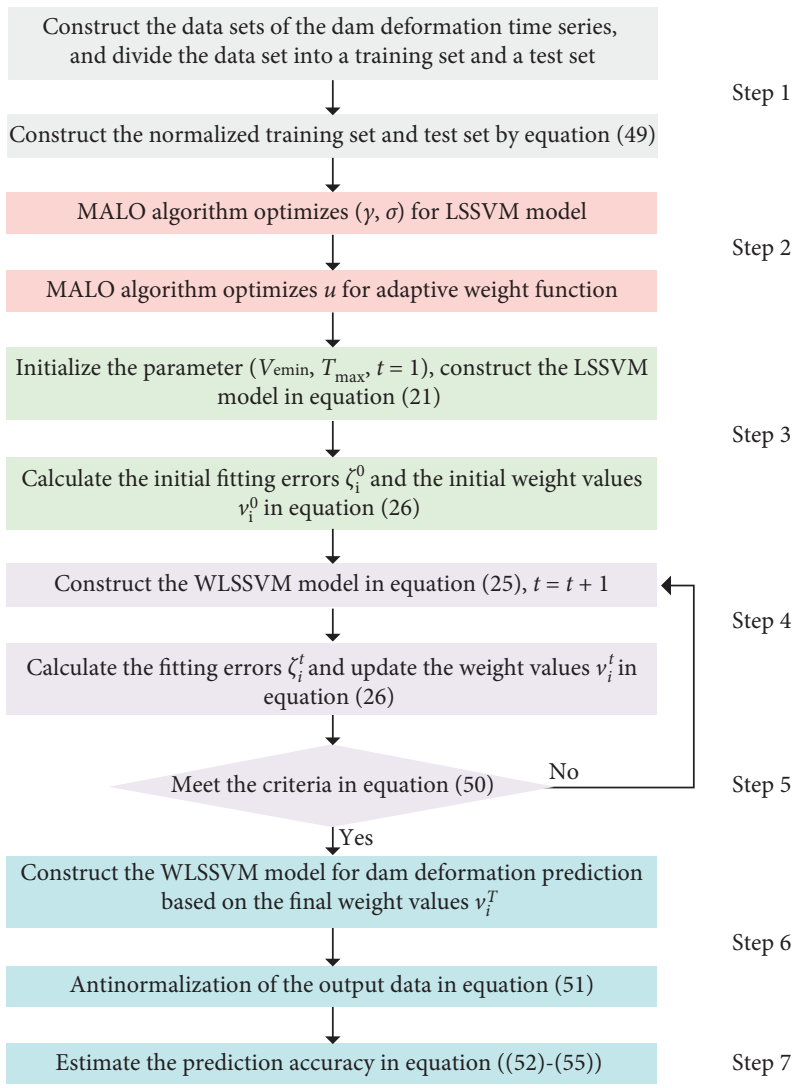

FIGURE 5: The construction process of the MALO-AWLSSVM model for predicting dam deformation.

be written as

$$
\begin{aligned}
\{\text { Input; Output }\} & =\left\{x_{1}, x_{2}, x_{3}, x_{4}, x_{5}, x_{6}, x_{7}, x_{8}, x_{9}, x_{10} ; y\right\} \\
& =\left\{H, H^{2}, H^{3}, H^{4}, \sin \frac{2 \pi t}{365}, \cos \frac{2 \pi t}{365}, \sin \frac{4 \pi t}{365}, \cos \frac{4 \pi t}{365}, \theta, \ln \theta ; y\right\} .
\end{aligned}
$$


To eliminate the effect of the index dimension and quantity of the data and to ensure that all the original data characteristics can be retained, all input data sets are normalized in the interval $[0,1]$ by equation

$$
x_{i}^{\prime}=\frac{x_{i}-x_{\min }}{x_{\max }-x_{\min }},
$$

where $x_{i}$ denotes the original data, $x_{\min }$ and $x_{\max }$ are the minimum value and maximum value of the original data, respectively, and $x_{i}^{\prime}$ represents the normalized data.

Step 2. Set parameters of the prediction model. The search space of punishment factor $\gamma$, kernel width $\sigma$, and adjustment coefficient $u$ are set as $[0.1,1000]$, [0.01, $10000]$, and $[0.1,10]$, respectively. The maximum iteration $t_{\max }$, the number of ants $N$, and the number of antlions $M$ are set as 100, 10, and 10, respectively; generate the first population of ants and antlions by the random sampling in the search space and the iteration step $t=0$. According to Section 4.3, conduct the MALO algorithm to optimize the punishment factor $\gamma$ and kernel width $\sigma$ for LSSVM model, and optimize the adjustment coefficient $u$ for adaptive weighted function in equation (26), and obtain the optimal combination of parameters $\left(\gamma_{\text {best }}, \sigma_{\text {best }}, u_{\text {best }}\right)$.

Step 3. Set the adaptive weighted iteration parameter; namely, $V_{e \min }, T_{\max }$ and $T$ are set to $0.0001,100$, and 1 , respectively; construct the LSSVM model by equation (21) based on the optimal combination of parameters $\left(\gamma_{\text {best }}, \sigma_{\text {best }}\right)$. The initial fitting errors $\xi_{i}^{0}\left(i=1,2, \ldots, N_{\text {train }}\right)$ of the training set can be obtained and the initial weight values $v_{i}^{0}\left(i=1,2, \ldots, N_{\text {train }}\right)$ can be calculated by equation (26) based on the optimal adjustment coefficient $u_{\text {best }}$. Step 4. Construct the WLSSVM model by equation (25) based on the weighted training set; calculate the fitting errors $\xi_{i}^{T}\left(i=1,2, \ldots, N_{\text {train }}\right)$ of the training set and update the corresponding weight values $v_{i}^{T}\left(i=1,2, \ldots, N_{\text {train }}\right)$ by equation (26).

Step 5. Suppose the termination criteria in equation (50) are satisfied; then output the weight values $v_{i}^{T}\left(i=1,2, \ldots, N_{\text {train }}\right)$; otherwise, update the iteration step $T=T+1$, and loop to step 4 .

$$
V_{e}=\frac{1}{N_{\text {train }}} \sum_{i=1}^{N_{\text {train }}}\left|v_{i}^{T}-v_{i}^{T-1}\right|<V_{e \min } \text { or } T>T_{\max } \text {. }
$$

Step 6. Establish the optimal WLSSVM prediction model according to the final weighted training set; implement the prediction with the optimal WLSSVM prediction model based on the test set, and obtain the normalized dam deformation value; then, the antinormalization of the output needs to be implemented by equation (51) to revert the dam deformation data.

$$
y_{i}^{*}=y_{i}^{*}\left(y_{\max }-y_{\min }\right)+y_{\min },
$$

where $y_{i}^{*}$ denotes the $i$-th predicted value, $y_{\min }$ and $y_{\max }$ are the minimum and maximum of the measured value, respectively, and $y_{i}^{*}$ represents the $i$-th normalized data of predicted value.

Step 7. Estimate the prediction accuracy of the MALOAWLSSVM model based on the test set. Four statistical indicators given in equations (52)-(55), the maximum error (ME), root mean squared error (RMSE), mean absolute error (MAE), and mean absolute percentage error (MAPE), are adopted to obtain an objective evaluation for the performance of the prediction models.

$$
\begin{aligned}
\mathrm{ME} & =\max _{1 \leq i \leq N}\left(\left|y_{i}-y_{i}^{*}\right|\right), \\
\mathrm{RMSE} & =\sqrt{\frac{1}{N} \sum_{i=1}^{N}\left(y_{i}-y_{i}^{*}\right)^{2},} \\
\mathrm{MAE} & =\frac{1}{N} \sum_{i=1}^{N}\left|y_{i}-y_{i}^{*}\right|, \\
\mathrm{MAPE} & =\frac{1}{N} \sum_{i=1}^{N} \frac{\left|y_{i}-y_{i}^{*}\right|}{\left|y_{i}\right|} \times 100 \%,
\end{aligned}
$$

where $N$ denotes the number of samples and $y_{i}$ and $y_{i}^{*}$ are the $i$-th measured value and predicted value, respectively.

\section{Case Study}

6.1. Engineering Overview and Dam Deformation Observation. The Jinping I concrete arch dam was selected as an example to validate the MALO-AWLSSVM prediction model, which is located on the Yalong River, Sichuan Province, China. The Yangtze River basin and the location of the Jinping I arch dam are shown in Figure 6. It mainly consists of a double-curvature thin arch dam, a power generation complex on the right bank, flood release structures, and diversion tunnels. The maximum height of the arch dam is $305.00 \mathrm{~m}$ (1580.00-1885.00 $\mathrm{m}$ asl), which is the highest arch dam in the world. The thickness of the dam crest is $16.00 \mathrm{~m}$, the base thickness is $63.00 \mathrm{~m}$, and the total arc length of the dam crest is $552.00 \mathrm{~m}$. The normal water level is $1880.00 \mathrm{~m}$, and the dead water level is $1800.00 \mathrm{~m}$. The total reservoir storage, flood regulation storage, and dead storage are $77.6 \times 10^{8}, 49.1 \times 10^{8}$, and $28.5 \times 10^{8} \mathrm{~m} 3$, respectively. The project was equipped with six Francis turbines, each rated at $600 \mathrm{MW}$ [42]. Figure 7 shows the "bird's eye" view and a central vertical cross section of the Jinping I arch dam.

The formal construction of this project was started on November 12, 2004, and, on December 4, 2006, river closure was completed. The dam in dam section 14 \# started pouring on October 23, 2009, and, on November 30, 2012, the arch dam was in a condition for reservoir impoundment in accordance with the fixed progress schedule. The arch dam was completed in December 2013. 


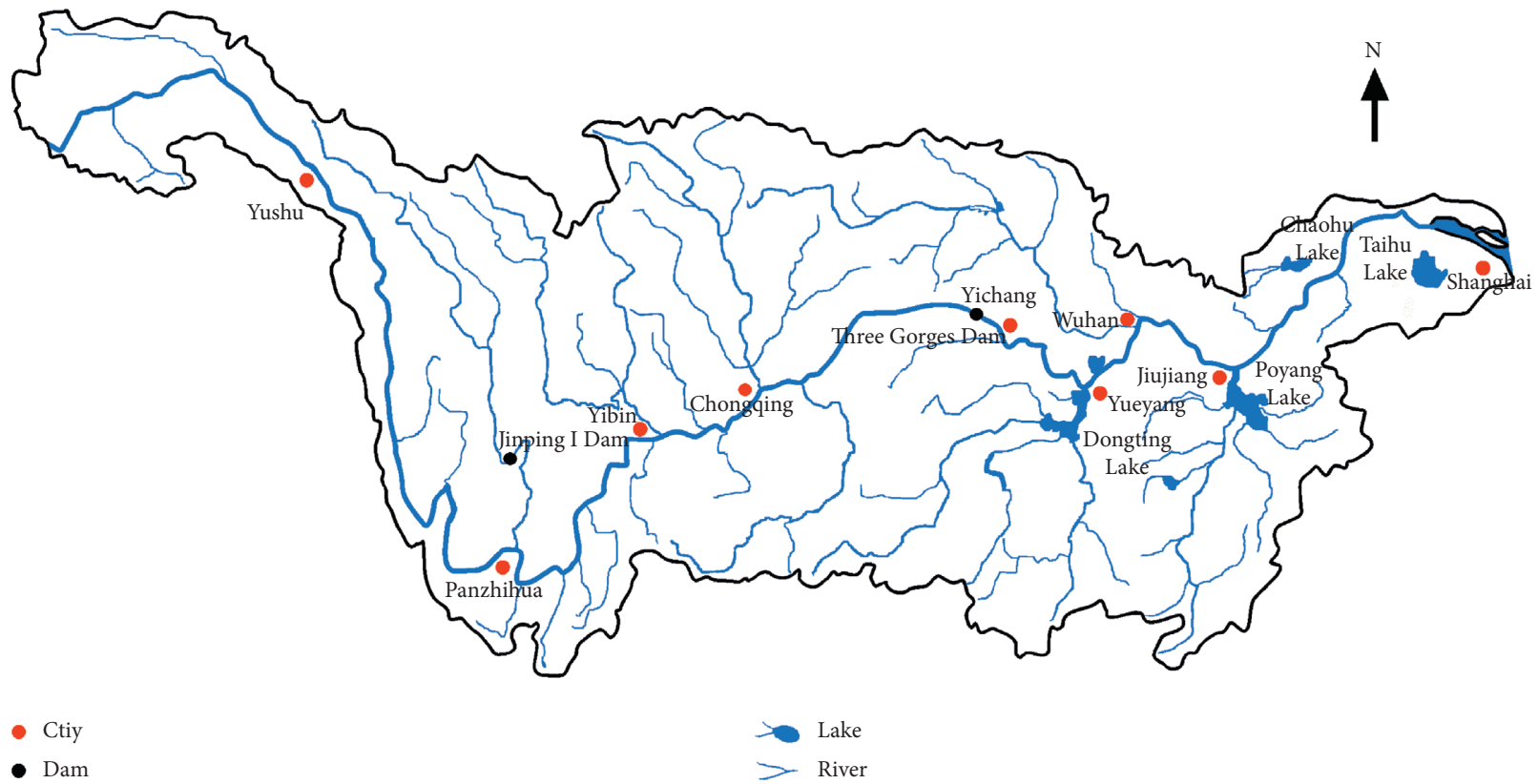

FIgURE 6: The Yangtze River basin and the location of the Jinping I arch dam.

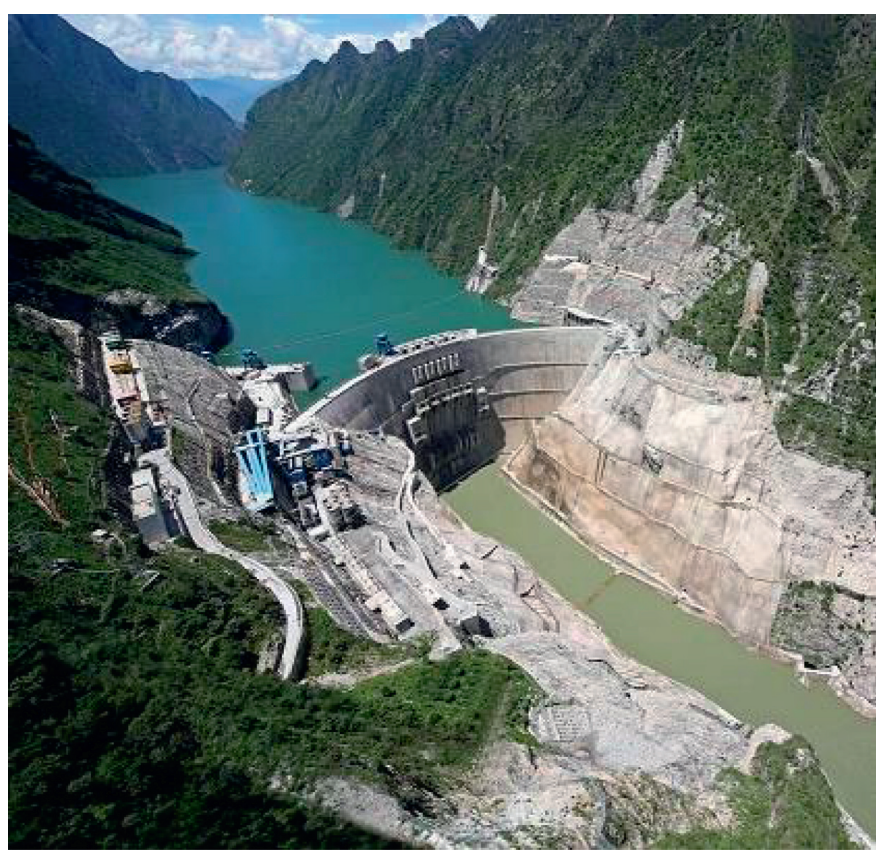

(a)

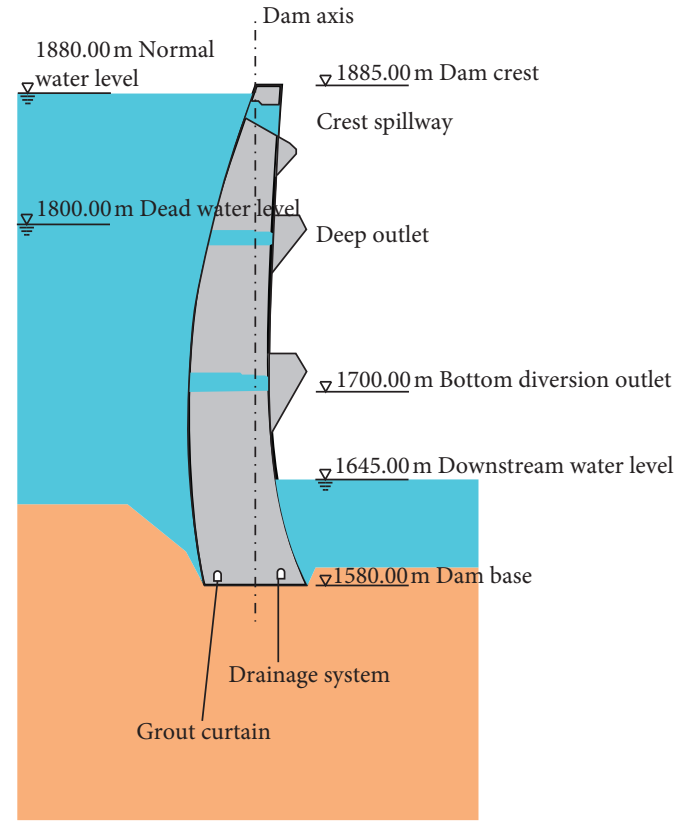

(b)

Figure 7: The Jinping I arch dam. (a) The "bird's eye" view; (b) central vertical cross section of the dam.

Figure 8 shows the arrangement diagram of the measuring points, which were installed to measure the horizontal displacement of the dam body. Figure 9 shows the schematic diagram of the plumb meter for monitoring the dam displacement. In this research, the radial displacement of measuring point PL13-4 in dam section 13\# from July 1, 2013, to December 31, 2018, was used as the study object. The progress line of the upstream water level and the measured radial displacement of PL13-4 are shown in Figure 10, resulting in a total of 1284 observations. The positive values denote the radial horizontal displacement downstream. The sequence is divided into a training set and a test set to verify the effectiveness of the proposed algorithm.

6.2. Comparison with Other Swarm Intelligence Algorithms. To demonstrate the improvements on the parameter optimization of the AWLSSVM model conducted by the MALO 


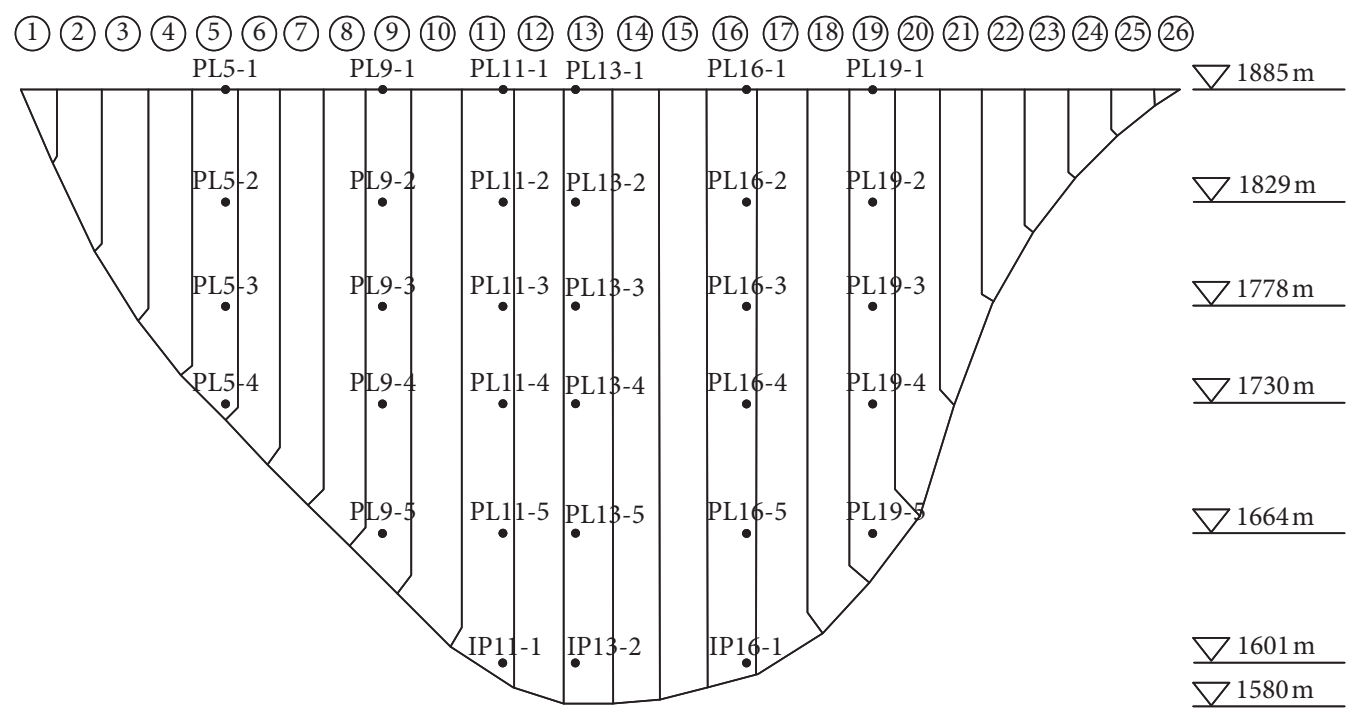

FIgURE 8: The layout of the pendulum measurements observing horizontal displacement.

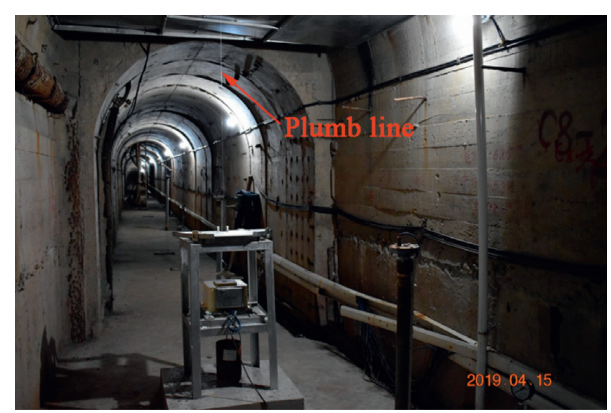

FIGURE 9: Schematic diagram of a plumb meter.

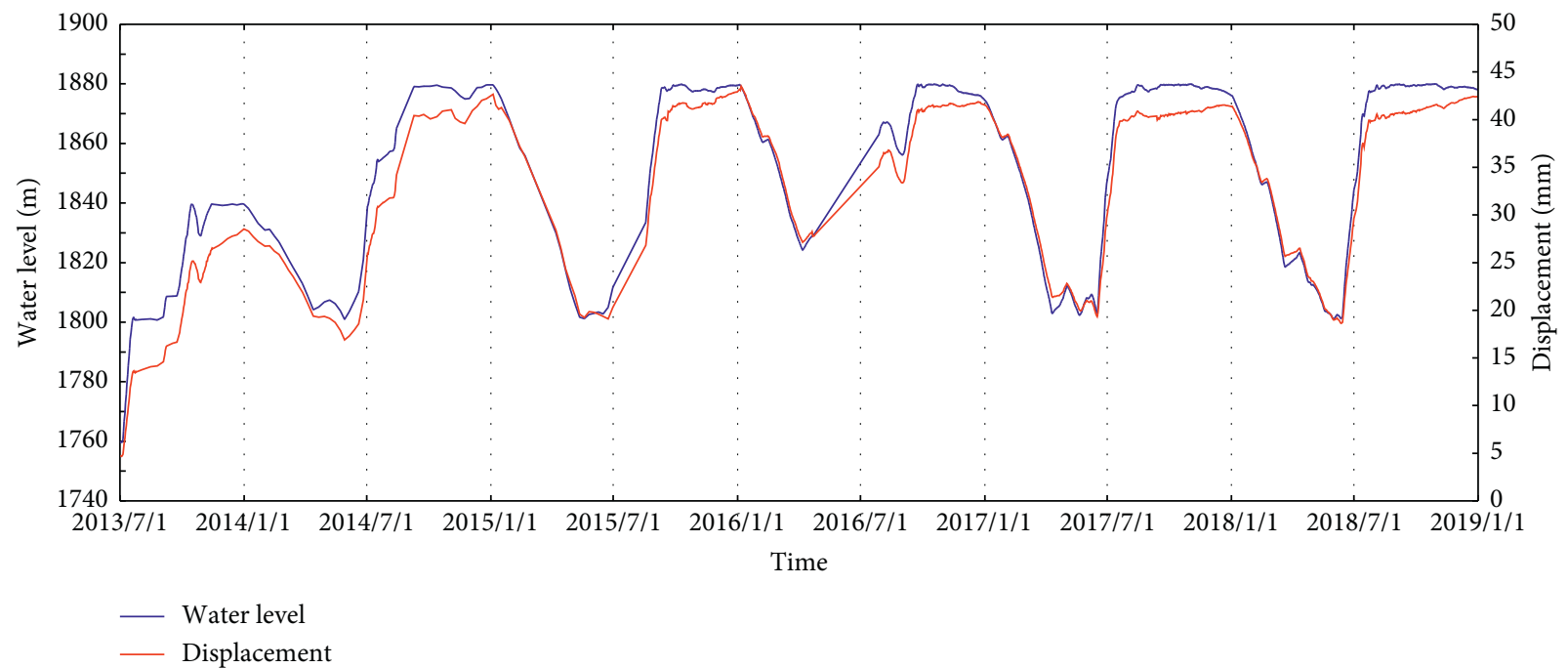

Figure 10: Time evolution of the water level and horizontal displacement at measuring point PL13-4.

algorithm, it was compared to other swarm intelligence algorithms such as the PSO [23], GSA [24], ACO [25], GWO [26], CS [27], Goa [28], SSA [29], and ALO models [30]. Table 2 lists the parameter settings of all algorithms used in this experiment. The punishment factor $\gamma$ and kernel width $\sigma$ of the LSSVM model and the adjustment coefficient $u$ in the adaptive weighted function were selected as shown in Table 3, which shows that the optimal parameters selected by different metaheuristic algorithms are close to some extent. Within that procedure, the four statistical indicators in 
TABLE 2: The parameter settings for the algorithms.

\begin{tabular}{|c|c|c|c|}
\hline Algorithms & Parameter & Explanation & Value \\
\hline \multirow{4}{*}{ PSO } & $N$ & Number of particles & 10 \\
\hline & $\omega_{\min }, \omega_{\max }$ & Minimum and maximum inertia weight & $0.4,0.8$ \\
\hline & $v_{\min }, v_{\max }$ & Minimum and maximum velocity & $-10,10$ \\
\hline & $c_{1}, c_{2}$ & Acceleration coefficients & $1.5,1.5$ \\
\hline \multirow{3}{*}{ GSA } & $N$ & Number of agents & 10 \\
\hline & $G_{0}$ & Initial value of the gravitational variable & 100 \\
\hline & $\alpha$ & User specified constant & 20 \\
\hline \multirow{3}{*}{$\mathrm{ACO}$} & $N$ & Number of ants & 10 \\
\hline & $\rho$ & Evaporation rate & 0.9 \\
\hline & $P_{0}$ & Transition probability constant & 0.2 \\
\hline \multirow{3}{*}{ GWO } & $N$ & Number of grey wolves & 10 \\
\hline & $a$ & Variable & {$[2,0]$} \\
\hline & $r_{1}, r_{2}$ & Random vectors & {$[0,1]$} \\
\hline \multirow{4}{*}{ CS } & $N$ & Number of host nests & 10 \\
\hline & $p_{a}$ & Detecting probability & 0.25 \\
\hline & $\alpha$ & Step size scaling factor & 0.01 \\
\hline & $\lambda$ & Levy index & 1.5 \\
\hline \multirow{4}{*}{ GOA } & $N$ & Number of grasshoppers & 10 \\
\hline & $c_{\min }, c_{\max }$ & Minimum and maximum value & $0.00004,1$ \\
\hline & $f$ & Intensity of attraction & 0.5 \\
\hline & $l$ & Attractive length scale & 1.5 \\
\hline \multirow{3}{*}{ SSA } & $N$ & Number of salps & 10 \\
\hline & $c_{1}$ & Variable & {$[2,0]$} \\
\hline & $c_{2}, c_{3}$ & Random number & {$[0,1]$} \\
\hline \multirow{3}{*}{ ALO } & $N$ & Number of ants & 10 \\
\hline & $M$ & Number of antlions & 10 \\
\hline & rand & Random number & {$[0,1]$} \\
\hline \multirow{4}{*}{ MALO } & $N$ & Number of ants & 10 \\
\hline & $M$ & Number of antlions & 10 \\
\hline & rand & Random number & {$[0,1]$} \\
\hline & $\lambda$ & Levy index & 1.5 \\
\hline
\end{tabular}

TABLE 3: Results of different swarm intelligence algorithms.

\begin{tabular}{|c|c|c|c|c|c|c|c|}
\hline Algorithms & $\gamma_{\text {best }}$ & $\sigma_{\text {best }}$ & $\mathbf{u}_{\text {best }}$ & $\mathrm{ME}(\mathrm{mm})$ & RMSE (mm) & $\operatorname{MAE}(\mathrm{mm})$ & MAPE (\%) \\
\hline PSO & 172 & 4437 & 3.29 & 0.311 & 0.148 & 0.126 & 0.387 \\
\hline GSA & 210 & 5407 & 3.25 & 0.312 & 0.148 & 0.126 & 0.388 \\
\hline $\mathrm{ACO}$ & 525 & 8995 & 3.61 & 0.428 & 0.214 & 0.174 & 0.506 \\
\hline GWO & 144 & 3724 & 3.31 & 0.311 & 0.149 & 0.127 & 0.387 \\
\hline CS & 145 & 3760 & 3.34 & 0.311 & 0.149 & 0.127 & 0.387 \\
\hline GOA & 358 & 9150 & 2.95 & 0.311 & 0.147 & 0.126 & 0.389 \\
\hline SSA & 168 & 4345 & 3.30 & 0.311 & 0.148 & 0.126 & 0.387 \\
\hline ALO & 266 & 6844 & 3.24 & 0.312 & 0.147 & 0.126 & 0.388 \\
\hline MALO & 266 & 6835 & 3.24 & 0.311 & 0.147 & 0.126 & 0.387 \\
\hline
\end{tabular}

equations (52)-(55) were applied to evaluate the prediction accuracy of each model, and the results are presented in Table 3. Table 3 shows that the MALOAWLSSVM model has a high prediction accuracy, and the prediction accuracy of the ACO-AWLSSVM model is the worst compared to that of the other models. Figure 11 displays the convergence procedure over the iterations of the ALO and MALO algorithms. The results indicate that the convergence rate of the MALO is faster than that of the ALO. Hence, the improved ALO algorithm can effectively improve the performance of the prediction model.
6.3. Stability Analysis of the Prediction Model by Introducing Different Numbers and Amplitudes of Outliers. In this study, to investigate the robust performance of the proposed model, different numbers and amplitudes of outliers are randomly added to the original training set. The definitions of the data sets with different outliers are listed in Table 4.

To compare the prediction performance, the MALOAWLSSVM, MALO-LSSVM, and multiple regression method (MRM) are applied to construct the prediction model based on the training set. Meanwhile, the test set is utilized to demonstrate their prediction performances for the models. Figure 12 displays the weight value distribution 


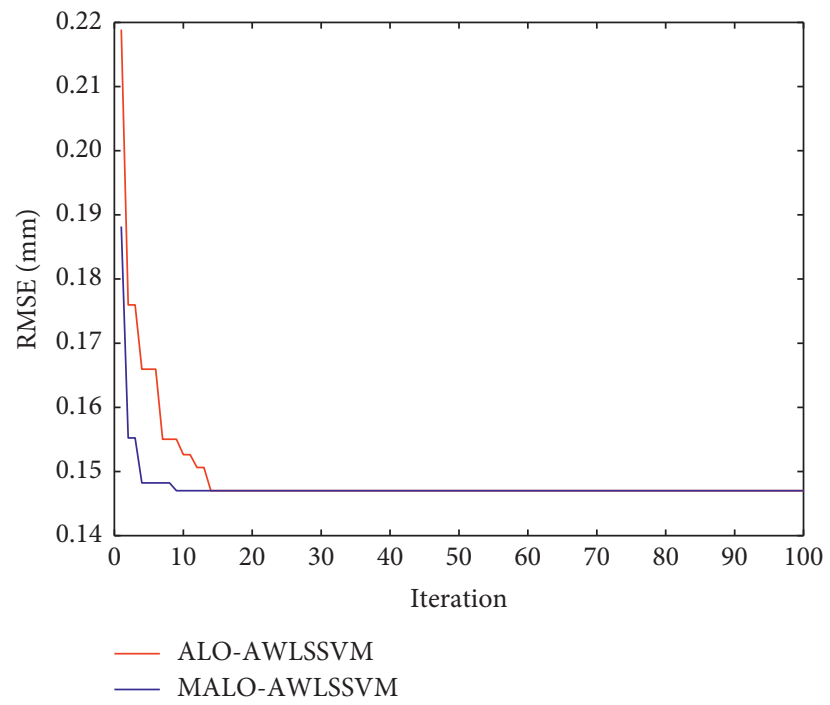

FIgURE 11: The convergence of the ALO-AWLSSVM and MALO-AWLSSVM algorithms.

TABLE 4: The definitions of the data sets with different outliers.

\begin{tabular}{|c|c|c|c|c|c|c|c|c|c|}
\hline Training set & Set 1 & Set 2 & Set 3 & Set 4 & Set 5 & Set 6 & Set 7 & Set 8 & Set 9 \\
\hline PER (\%) & 0 & 1 & 1 & 1 & 1 & 2 & 2 & 2 & 2 \\
\hline $\operatorname{AMP}(\mathrm{mm})$ & 0 & 1 & 2 & 3 & 4 & 1 & 2 & 3 & 4 \\
\hline Training set & Set 10 & Set 11 & Set 12 & Set 13 & Set 14 & Set 15 & Set 16 & Set 17 & - \\
\hline PER (\%) & 3 & 3 & 3 & 3 & 4 & 4 & 4 & 4 & - \\
\hline $\operatorname{AMP}(\mathrm{mm})$ & 1 & 2 & 3 & 4 & 1 & 2 & 3 & 4 & - \\
\hline
\end{tabular}

Note: PER represents the ratio of the number of outliers to the number of the training sets, AMP denotes the absolute amplitude of the outlier, and the ratio of the test set to the data set is $25 \%$.

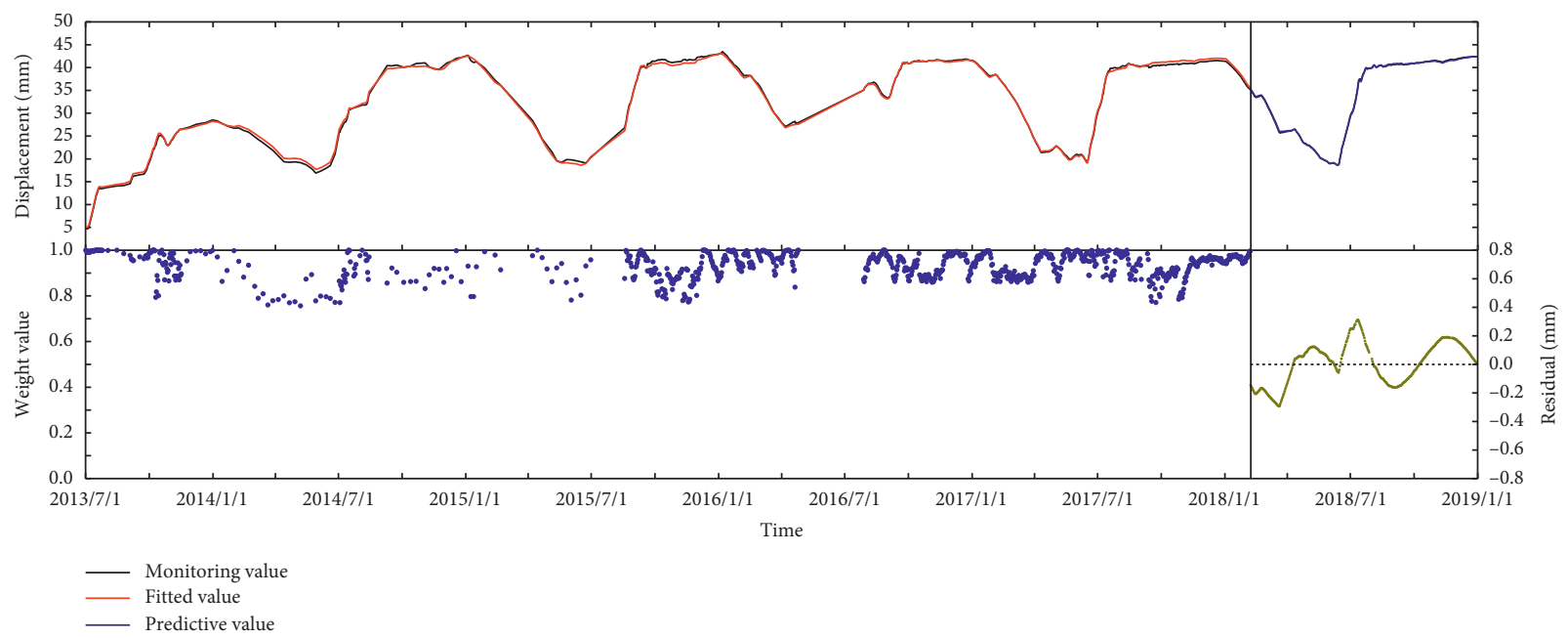

(a)

FIGURE 12: Continued. 


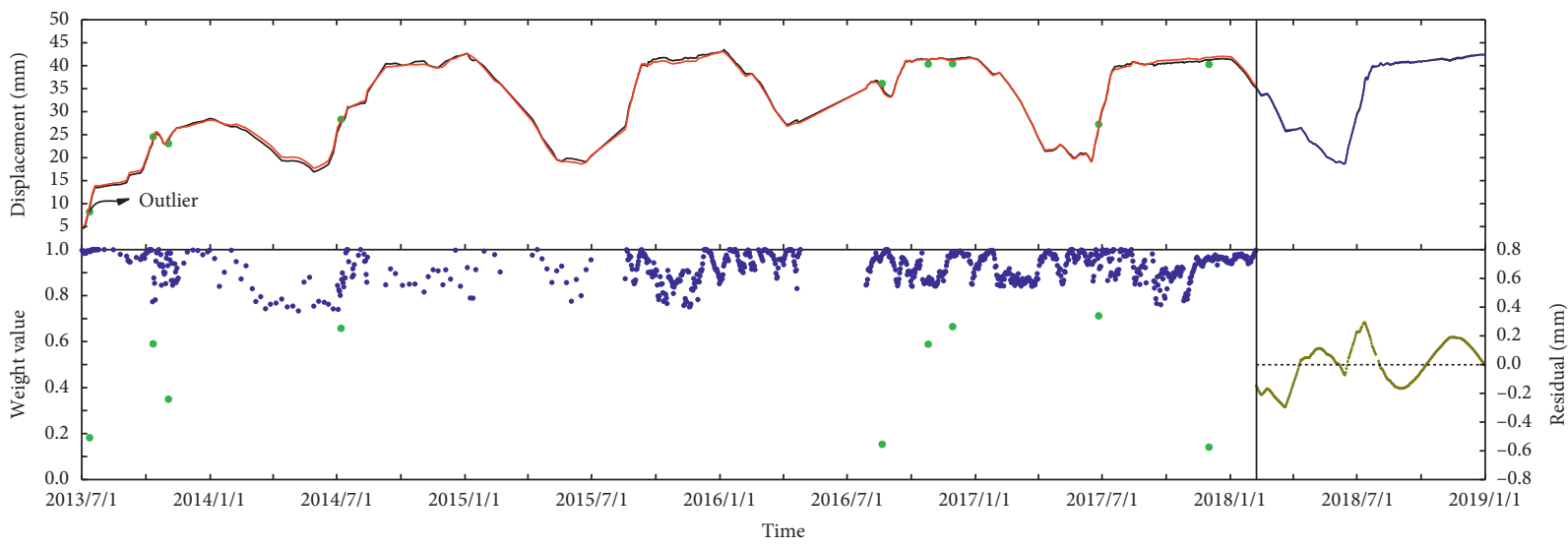

_ Monitoring value

__ Fitted value

_ Predictive value

(b)

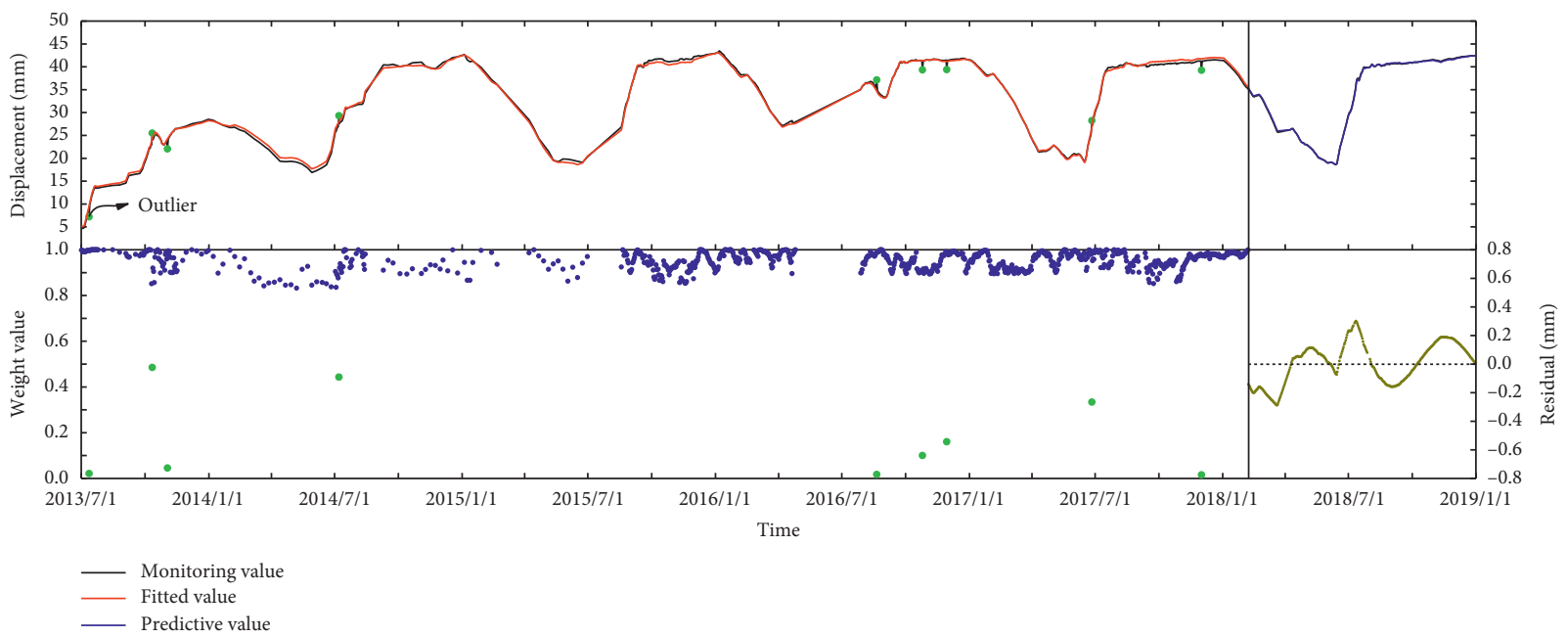

(c)

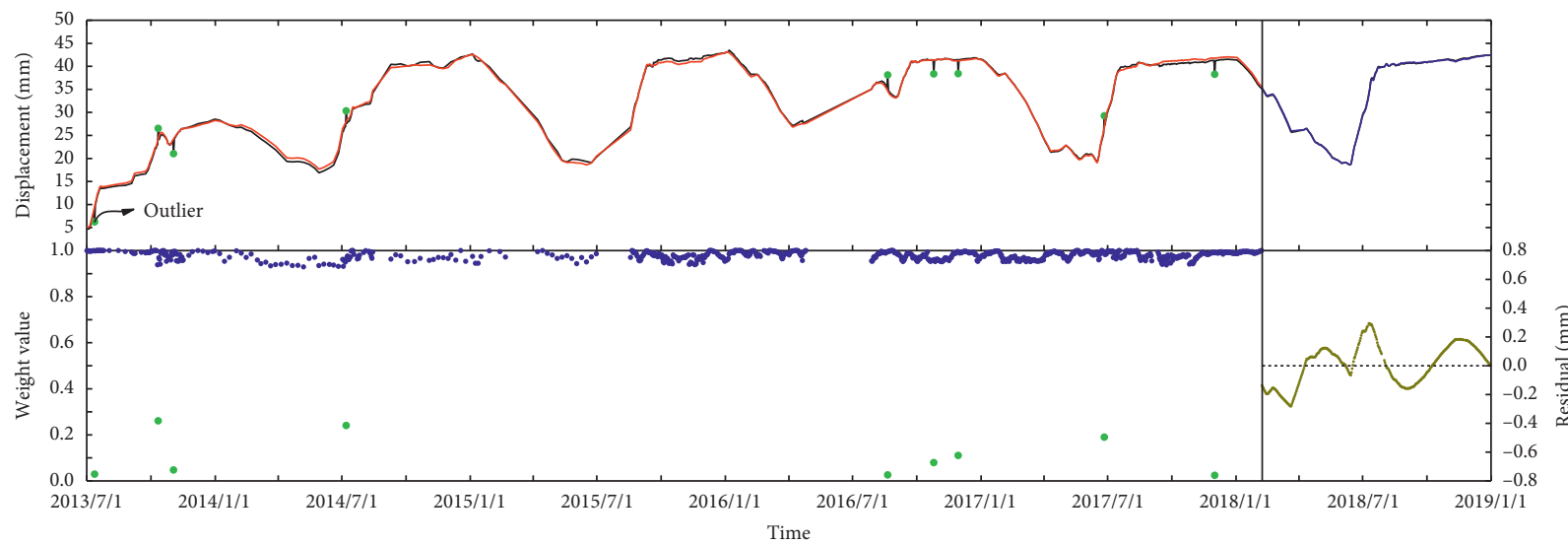

(d)

Figure 12: Continued. 


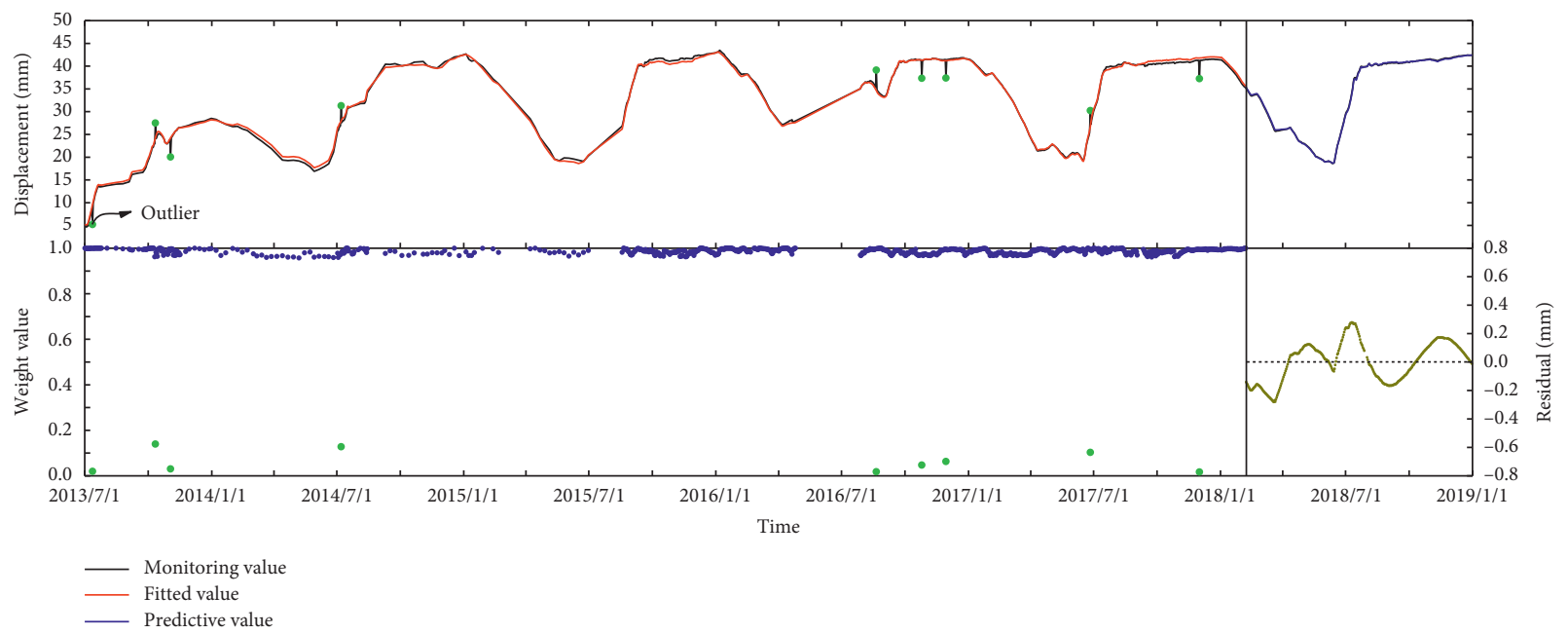

(e)

FIgURE 12: The weight value distribution of the training set and the prediction results of the MALO-AWLSSVM model. (a) Set 1; (b) set 2; (c) set 3 ; (d) set 4 ; (e) set 5 .

PER

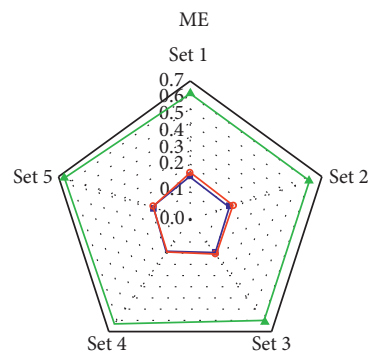

$2 \%$

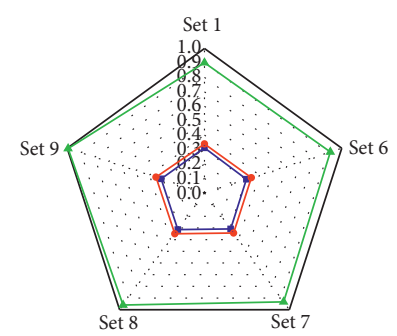

$3 \%$

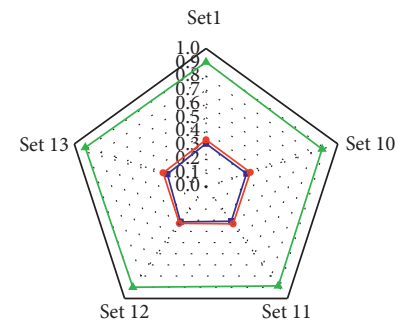

$4 \%$

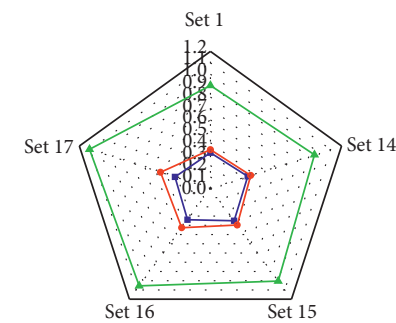

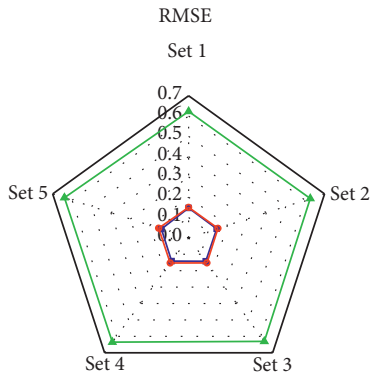

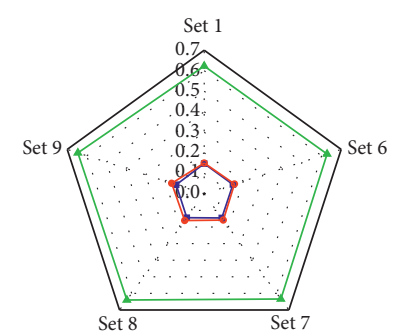

Set 1

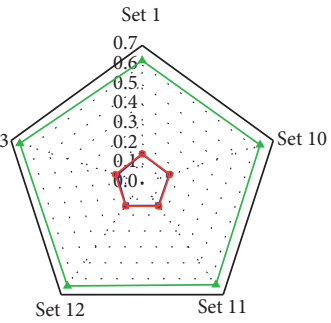

Set 1

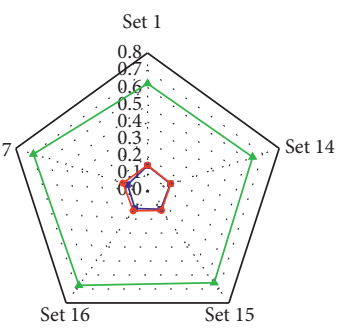

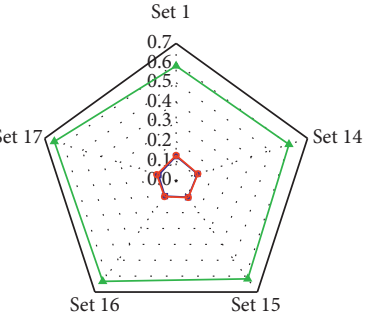

MAPE
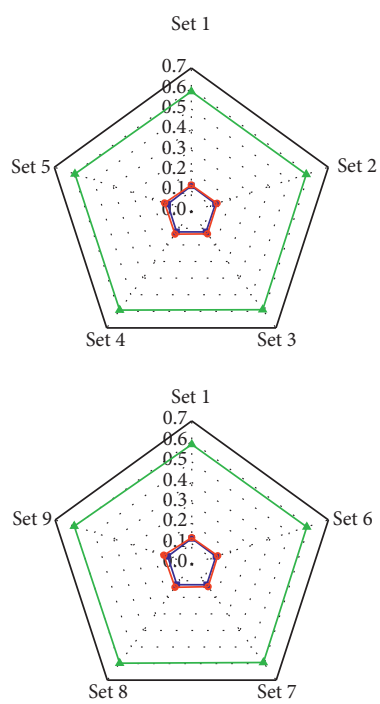

Set 1
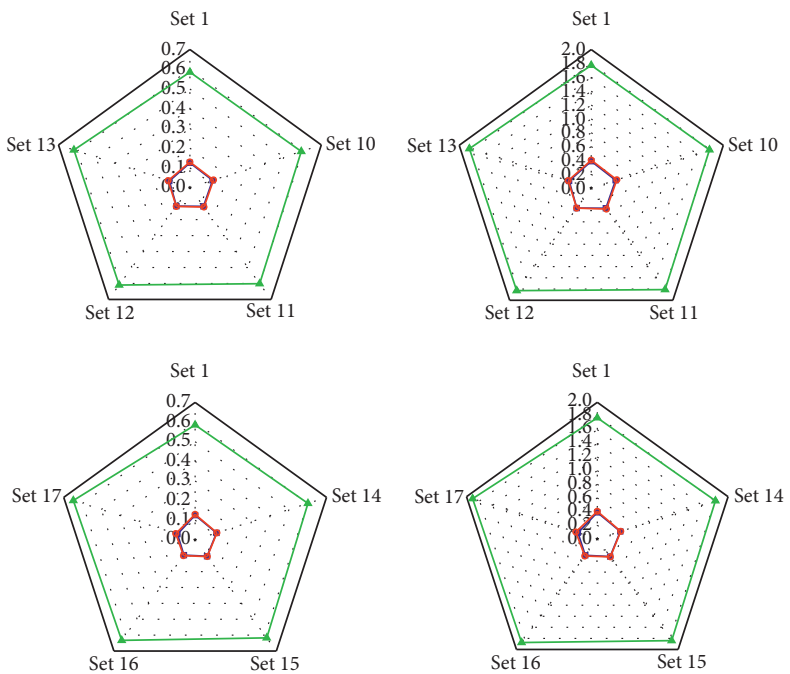

Figure 13: Comparison of the prediction performance using three prediction models in different data sets. The blue line represents the MALO-AWLSSVM model, the red line denotes the MALO-LSSVM model, and the green line is the MRM model. 
TABle 5: Performance indicators (ME, RMSE, MAE, and MAPE) values using three prediction models for dam deformation prediction in different data sets.

\begin{tabular}{|c|c|c|c|c|c|c|c|c|c|c|c|c|}
\hline \multirow{2}{*}{ Data sets } & \multicolumn{4}{|c|}{ MALO-AWLSSVM } & \multicolumn{4}{|c|}{ MALO-LSSVM } & \multicolumn{4}{|c|}{ MRM } \\
\hline & ME & RMSE & MAE & MAPE & $\mathrm{ME}$ & RMSE & MAE & MAPE & ME & RMSE & MAE & MAPE \\
\hline Set 1 & 0.311 & 0.147 & 0.126 & 0.387 & 0.339 & 0.150 & 0.130 & 0.405 & 0.902 & 0.623 & 0.585 & 1.774 \\
\hline Set 2 & 0.296 & 0.145 & 0.124 & 0.379 & 0.326 & 0.151 & 0.132 & 0.407 & 0.896 & 0.627 & 0.588 & 1.779 \\
\hline Set 3 & 0.301 & 0.143 & 0.122 & 0.374 & 0.310 & 0.153 & 0.134 & 0.409 & 0.911 & 0.631 & 0.590 & 1.784 \\
\hline Set 4 & 0.294 & 0.142 & 0.122 & 0.375 & 0.296 & 0.153 & 0.135 & 0.412 & 0.931 & 0.635 & 0.593 & 1.789 \\
\hline Set 5 & 0.281 & 0.140 & 0.120 & 0.372 & 0.281 & 0.155 & 0.137 & 0.415 & 0.952 & 0.640 & 0.595 & 1.795 \\
\hline Set 6 & 0.306 & 0.147 & 0.124 & 0.379 & 0.342 & 0.155 & 0.133 & 0.409 & 0.917 & 0.628 & 0.590 & 1.786 \\
\hline Set 7 & 0.308 & 0.145 & 0.124 & 0.379 & 0.343 & 0.158 & 0.136 & 0.416 & 0.933 & 0.634 & 0.594 & 1.799 \\
\hline Set 8 & 0.314 & 0.144 & 0.123 & 0.382 & 0.350 & 0.161 & 0.139 & 0.432 & 0.960 & 0.640 & 0.598 & 1.812 \\
\hline Set 9 & 0.316 & 0.143 & 0.123 & 0.381 & 0.353 & 0.166 & 0.143 & 0.443 & 0.994 & 0.646 & 0.602 & 1.825 \\
\hline Set 10 & 0.307 & 0.144 & 0.121 & 0.369 & 0.336 & 0.148 & 0.126 & 0.388 & 0.881 & 0.630 & 0.593 & 1.792 \\
\hline Set 11 & 0.309 & 0.140 & 0.117 & 0.359 & 0.332 & 0.145 & 0.121 & 0.372 & 0.888 & 0.637 & 0.602 & 1.810 \\
\hline Set 12 & 0.314 & 0.141 & 0.116 & 0.355 & 0.328 & 0.143 & 0.117 & 0.356 & 0.899 & 0.645 & 0.610 & 1.828 \\
\hline Set 13 & 0.314 & 0.141 & 0.116 & 0.355 & 0.328 & 0.143 & 0.117 & 0.356 & 0.899 & 0.645 & 0.610 & 1.828 \\
\hline Set 14 & 0.341 & 0.139 & 0.114 & 0.352 & 0.369 & 0.142 & 0.116 & 0.359 & 0.952 & 0.639 & 0.601 & 1.807 \\
\hline Set 15 & 0.351 & 0.130 & 0.103 & 0.318 & 0.397 & 0.138 & 0.105 & 0.325 & 1.004 & 0.656 & 0.617 & 1.840 \\
\hline Set 16 & 0.339 & 0.124 & 0.096 & 0.297 & 0.426 & 0.141 & 0.100 & 0.310 & 1.056 & 0.674 & 0.633 & 1.874 \\
\hline Set 17 & 0.324 & 0.121 & 0.094 & 0.296 & 0.457 & 0.149 & 0.103 & 0.328 & 1.107 & 0.693 & 0.649 & 1.907 \\
\hline
\end{tabular}

TABLE 6: The definitions of the data sets with different outliers and different lengths of the test set.

\begin{tabular}{lcccccccc}
\hline $\begin{array}{l}\text { Training } \\
\text { set }\end{array}$ & $\begin{array}{c}\text { Set } \\
1\end{array}$ & Set 2 & Set 3 & Set 4 & Set 5 & Set 6 & Set 7 & $\begin{array}{c}\text { Set } \\
8\end{array}$ \\
\hline PRL (\%) & 10 & 10 & 10 & 10 & 10 & 20 & 20 & 20 \\
PER (\%) & 0 & 1 & 2 & 3 & 4 & 0 & 1 & 2 \\
\hline $\begin{array}{l}\text { Training } \\
\text { set }\end{array}$ & Set 9 & Set 10 & Set 11 & Set 12 & Set 13 & Set 14 & Set 15 & - \\
\hline PRL (\%) & 20 & 20 & 30 & 30 & 30 & 30 & 30 & - \\
PER (\%) & 3 & 4 & 0 & 1 & 2 & 3 & 4 & - \\
\hline
\end{tabular}

Note: PRL represents the ratio of the test set to the data set, PER represents the ratio of the number of outliers to the number of the training sets, and the absolute amplitude of the outlier is $3 \mathrm{~mm}$.

of the training set and the prediction results of the proposed model. Figure 12 shows that the weight values corresponding to the outlier are smaller than those of other normal measuring points. The trend of the residual variables is consistent in different data sets. In this way, the influence of the outliers on the model performance can be effectively reduced, and the stability and prediction accuracy of the model can be improved.

At the same time, the performance indicators (ME, RMSE, MAE, and MAPE) defined by equations (52)-(55) are applied to quantitatively estimate the prediction performance of the three models. The prediction accuracy of different models in different data sets is shown in Figure 13, and the performance indicator values are listed in Table 5. It can be clearly concluded that the MALO-AWLSSVM model and MALO-LSSVM model both have good fitting accuracy, but the MALO-AWLSSVM model is better, and the same performance indicators are larger for the multiple regression model. For the MALO-AWLSSVM model, the prediction performance is almost unaffected by outliers in the training set. As the number and amplitude of outliers increase, the prediction performance of the proposed model is better than those of the other two prediction models. By weighting each sample in the training set, the MALO-AWLSSVM model can effectively eliminate the influence of outliers and has stronger robustness. When the number of outliers is the same and the amplitude is different, the prediction performance of the three models is relatively stable.

6.4. Stability Analysis of the Prediction Model by Introducing Different Amounts of Outliers and Different Lengths of the Test Set. For the second scheme, different numbers of outliers are randomly added to the origin training set, and different lengths of the test sets are investigated. The definitions of the data sets with different outliers and different lengths of the test set are listed in Table 6.

To compare the prediction performance, MALOAWLSSVM, MALO-LSSVM, and multiple regression method (MRM) are applied to establish the prediction model based on the training set. Meanwhile, the test set is utilized to demonstrate the predictive performance of the models. As seen from Figure 14, the distribution of the outliers in the training set can be clearly identified based on the weight value. The trends of the residual variables are consistent in the data sets with different numbers of outliers. In conclusion, the MALO-AWLSSVM model has a very stable prediction performance and effectively eliminates the influence of outliers on the prediction accuracy.

Meanwhile, the performance indicators (ME, RMSE, MAE, and MAPE) by equations (52)-(55) are applied to evaluate the prediction performance of the three models. The prediction accuracy of the different models for different data sets is shown in Figure 15, and the performance indicator values are listed in Table 7. For the same data set, the MALOAWLSSVM model has the best prediction performance, and the MRM is the worst. As the number of outliers increases, the prediction performance of the three models is relatively 


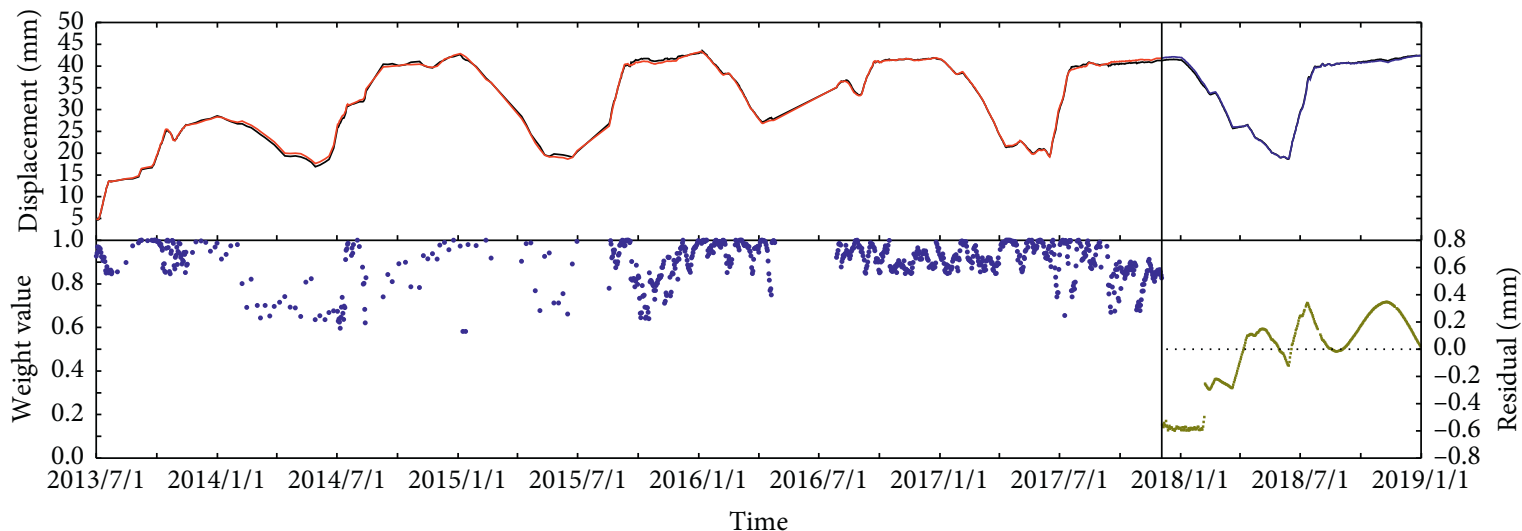

— Monitoring value

Fitted value

Predictive value

(a)

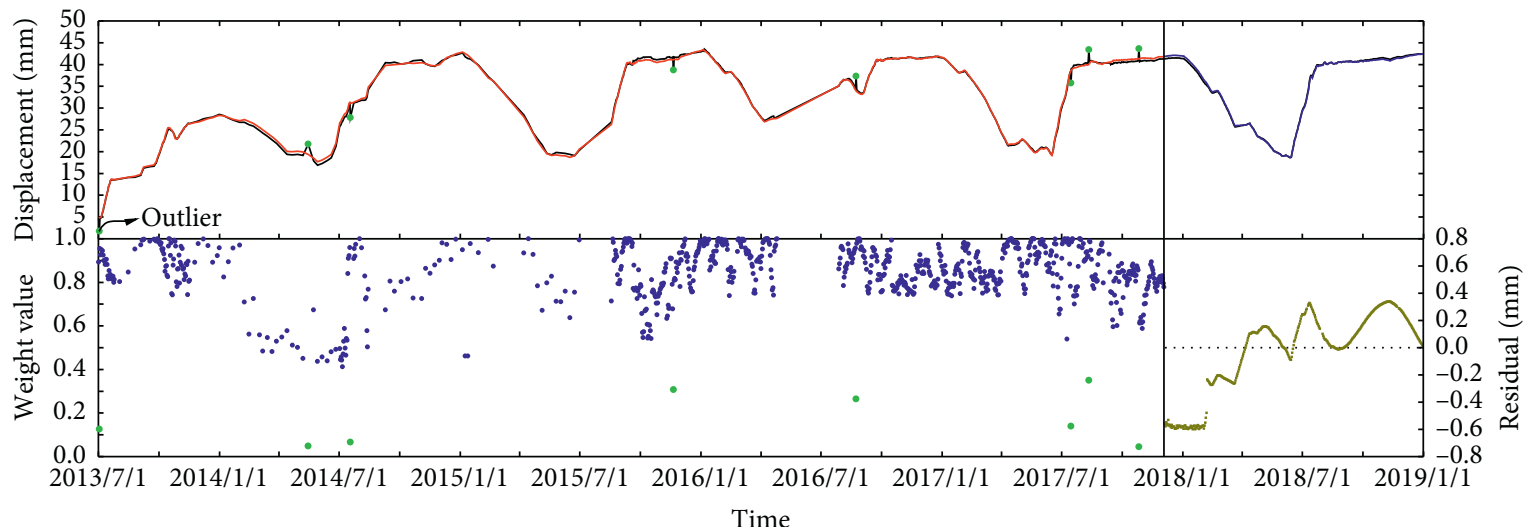

- Monitoring value

Fitted value

Predictive value

(b)

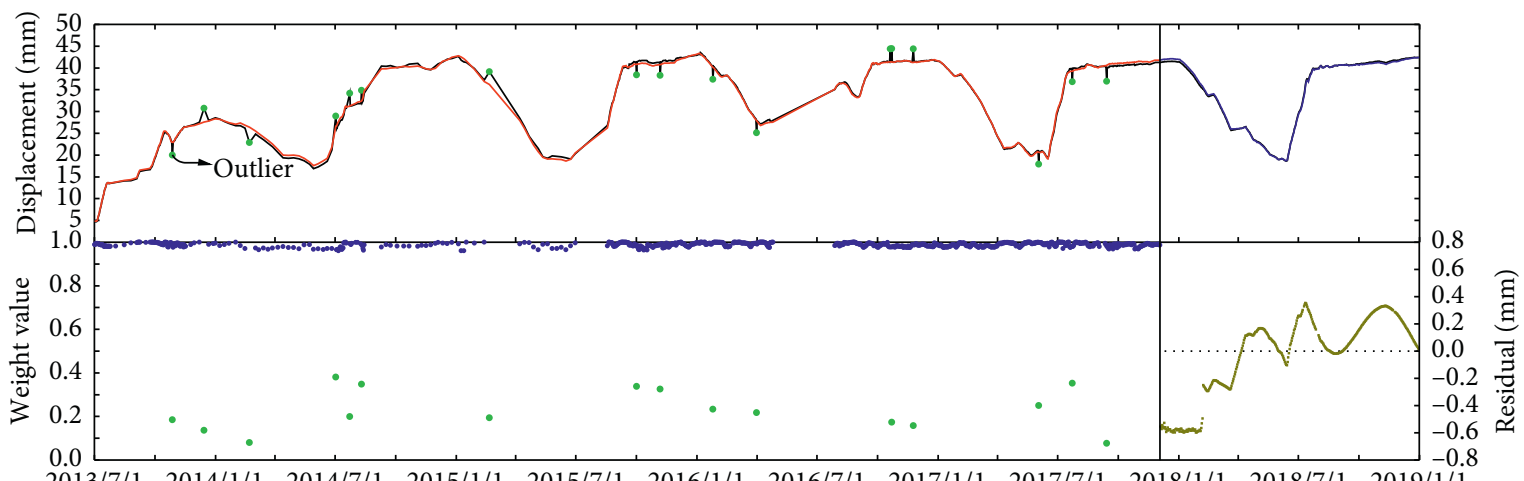

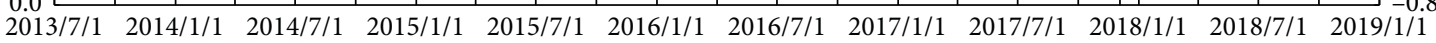

Time

Monitoring value

Fitted value

Predictive value

(c)

Figure 14: Continued. 


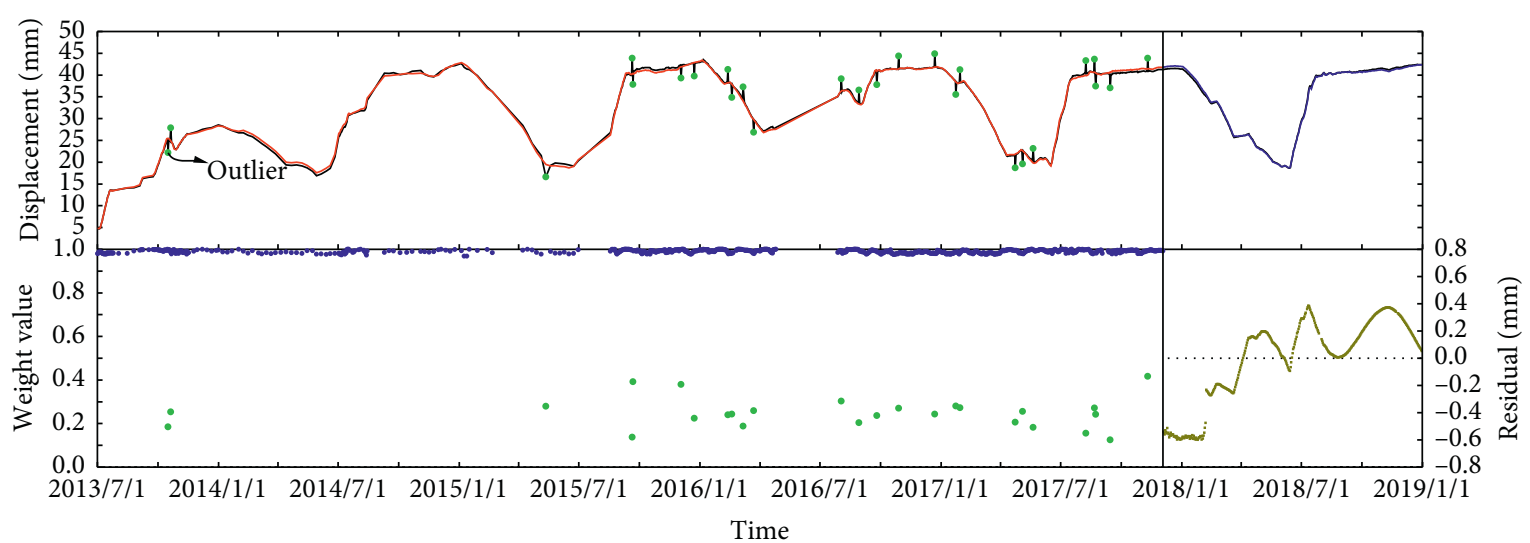

_ Monitoring value

— Fitted value

Predictive value

(d)

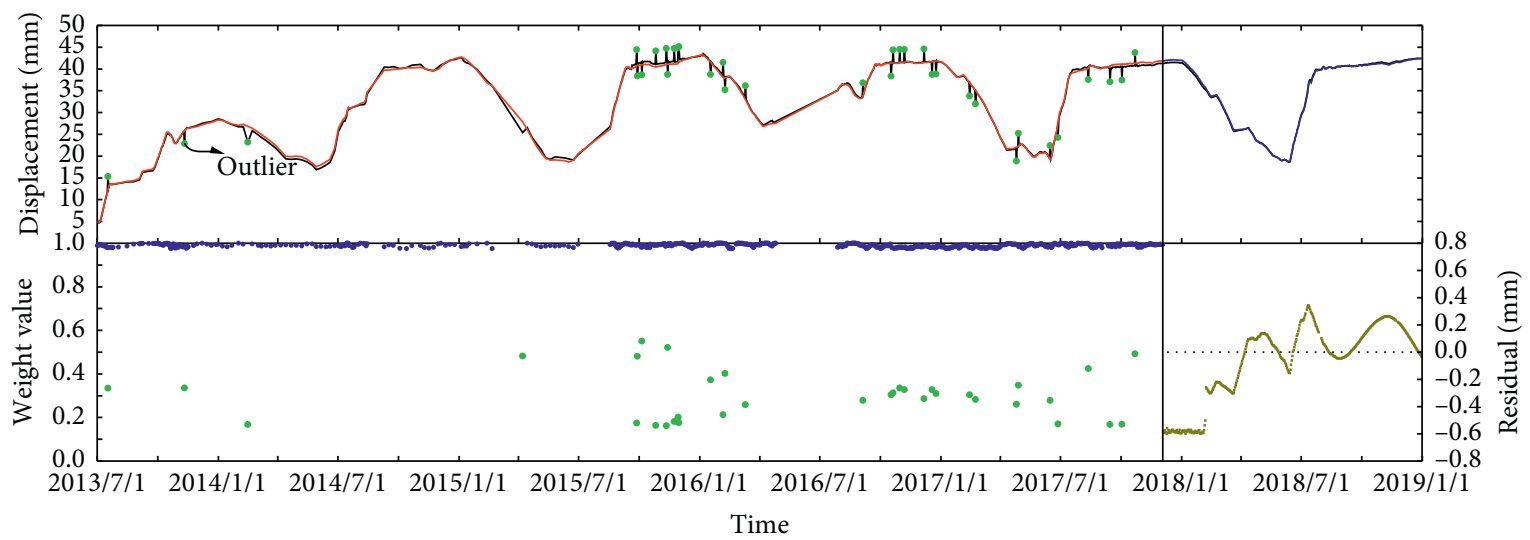

Monitoring value
_ Fitted value
_ Predictive value

(e)

FIGURE 14: The weight value distribution of the training set and the prediction results of the MALO-AWLSSVM model. (a) Set 11; (b) set 12; (c) set 13 ; (d) set 14 ; (e) set 15 .

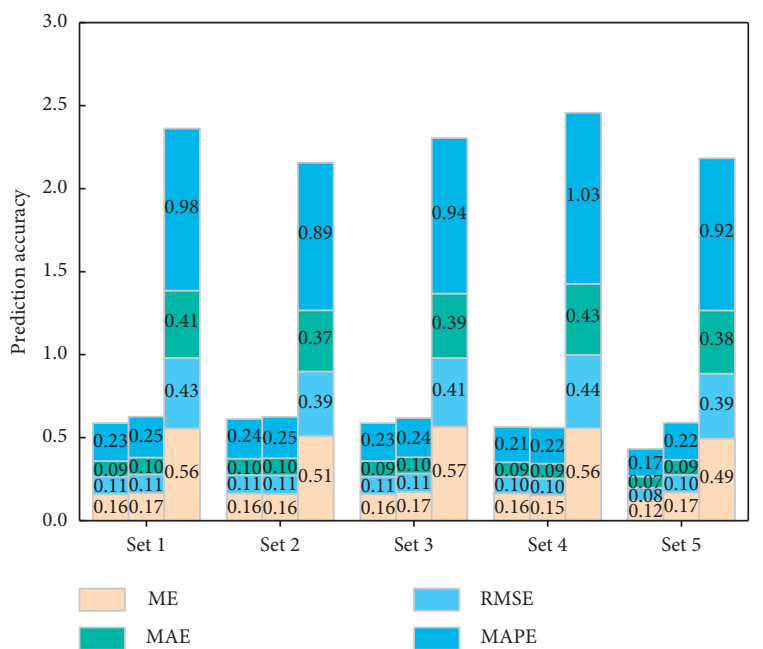

(a)

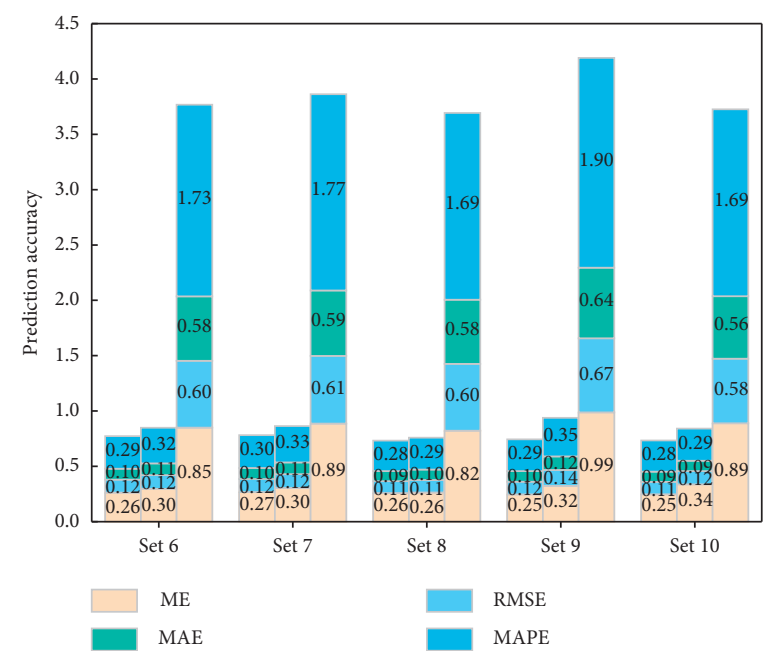

(b)

Figure 15: Continued. 


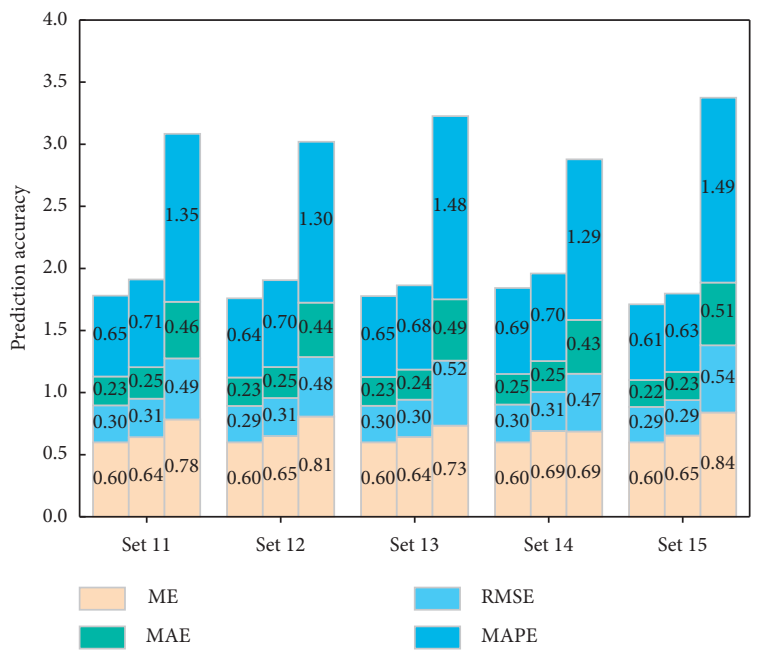

(c)

FIGURE 15: Comparison of the prediction performance using three prediction models in different data sets (for each group, the left column represents the MALO-AWLSSVM model, the middle column denotes the MALO-LSSVM model, and the right column is the MRM model). (a) $\mathrm{PRL}=10 \%$; (b) $\mathrm{PRL}=20 \%$; (c) $\mathrm{PRL}=30 \%$.

TABLE 7: Performance indicators (ME, RMSE, MAE, and MAPE) values using three prediction models for dam deformation prediction in different data sets.

\begin{tabular}{|c|c|c|c|c|c|c|c|c|c|c|c|c|}
\hline \multirow{2}{*}{ Data sets } & \multicolumn{4}{|c|}{ MALO-AWLSSVM } & \multicolumn{4}{|c|}{ MALO-LSSVM } & \multicolumn{4}{|c|}{ MRM } \\
\hline & $\mathrm{ME}$ & RMSE & MAE & MAPE & $\mathrm{ME}$ & RMSE & MAE & MAPE & ME & RMSE & MAE & MAPE \\
\hline Set 1 & 0.159 & 0.106 & 0.094 & 0.228 & 0.165 & 0.114 & 0.102 & 0.246 & 0.555 & 0.426 & 0.405 & 0.976 \\
\hline Set 2 & 0.164 & 0.111 & 0.098 & 0.239 & 0.161 & 0.113 & 0.102 & 0.248 & 0.510 & 0.388 & 0.369 & 0.888 \\
\hline Set 3 & 0.159 & 0.106 & 0.094 & 0.228 & 0.173 & 0.111 & 0.097 & 0.236 & 0.567 & 0.413 & 0.389 & 0.936 \\
\hline Set 4 & 0.164 & 0.101 & 0.087 & 0.212 & 0.153 & 0.102 & 0.090 & 0.217 & 0.557 & 0.441 & 0.427 & 1.030 \\
\hline Set 5 & 0.120 & 0.077 & 0.069 & 0.166 & 0.171 & 0.105 & 0.092 & 0.223 & 0.495 & 0.391 & 0.380 & 0.917 \\
\hline Set 6 & 0.263 & 0.117 & 0.099 & 0.294 & 0.297 & 0.124 & 0.106 & 0.322 & 0.850 & 0.604 & 0.583 & 1.731 \\
\hline Set 7 & 0.268 & 0.118 & 0.100 & 0.296 & 0.304 & 0.125 & 0.106 & 0.328 & 0.886 & 0.611 & 0.591 & 1.774 \\
\hline Set 8 & 0.259 & 0.107 & 0.092 & 0.275 & 0.264 & 0.112 & 0.096 & 0.287 & 0.823 & 0.603 & 0.580 & 1.687 \\
\hline Set 9 & 0.246 & 0.115 & 0.096 & 0.286 & 0.324 & 0.144 & 0.122 & 0.349 & 0.988 & 0.668 & 0.638 & 1.896 \\
\hline Set 10 & 0.247 & 0.112 & 0.093 & 0.281 & 0.338 & 0.119 & 0.093 & 0.291 & 0.889 & 0.584 & 0.564 & 1.689 \\
\hline Set 11 & 0.600 & 0.297 & 0.233 & 0.651 & 0.641 & 0.309 & 0.254 & 0.707 & 0.783 & 0.492 & 0.456 & 1.353 \\
\hline Set 12 & 0.600 & 0.293 & 0.229 & 0.638 & 0.650 & 0.307 & 0.249 & 0.700 & 0.807 & 0.479 & 0.439 & 1.296 \\
\hline Set 13 & 0.599 & 0.295 & 0.232 & 0.651 & 0.643 & 0.301 & 0.242 & 0.678 & 0.734 & 0.523 & 0.493 & 1.476 \\
\hline Set 14 & 0.599 & 0.304 & 0.247 & 0.692 & 0.691 & 0.314 & 0.249 & 0.705 & 0.687 & 0.465 & 0.434 & 1.293 \\
\hline Set 15 & 0.600 & 0.285 & 0.216 & 0.610 & 0654 & 0.286 & 0.226 & 0.632 & 0.839 & 0.541 & 0.505 & 1.489 \\
\hline
\end{tabular}

stable. When the length of the test set is larger, the prediction performance of the three models is worse compared to that when the test set is smaller. As the prediction ratio PRL increases from $20 \%$ to $30 \%$, the performance indicator values of the MALO-AWLSSVM model and the MALOLSSVM model increase more significantly. This could be because the LSSVM model relies on more training data to obtain a better prediction model. Nevertheless, in terms of the prediction performance, the MALO-AWLSSVM model is superior to the other two models.

\section{Conclusions}

The problems associated with dam deformation prediction will become more complicated and challenging with the development of the social economy and the effects of extreme climates. As a result, dam deformation prediction requires further attention and efficient prediction techniques. In this study, a novel dam deformation prediction strategy inspired by a regression model and a novel natureinspired metaheuristic algorithm is proposed. The conclusions obtained are discussed as follows:

(1) The current study combined AWLSSVM model and the MALO algorithm to predict the dam deformation. To avoid the blindness of parameter selection and improve the prediction performance of the prediction model, the MALO algorithm was used to intelligently select the punishment factor and kernel width in the LSSVM model and the adjustment coefficient in the Gaussian adaptive weighting 
function. Additionally, the advantages of the MALO algorithm were compared with other metaheuristic algorithms such as the PSO, GSA, ACO, GWO, CS, GOA, SSA, and ALO algorithm models. The results revealed that the MALO algorithm has high prediction accuracy, and the convergence rate is faster than that of the ALO. Hence, the MALO algorithm is a more reliable algorithm to effectively conduct parameter optimization.

(2) An actual dam project was employed to benchmark the performance of the MALO-AWLSSVM model. To explore the fitting and prediction effect of the model, we set two schemes for the data set. For the first scheme, the training sets were introduced with different numbers and amplitudes of the outliers. The second scheme was based on considering different numbers of outliers and different lengths of the test sets. An analysis of the dam deformation was carried out under the framework of each data set. Meanwhile, the other different prediction models (i.e., the MALO-LSSVM model and MRM model) were employed to provide the comparison. Among these prediction models, the statistical indicators demonstrated that the MALO-AWLSSVM model has a better performance in fitting ability and prediction accuracy, which has certain guiding significance for dam deformation prediction. The results showed that the Gaussian weighting strategy introduced to adaptively weight the training sample datum is a successful technique that can effectively eliminate the influence of outliers and achieve highprecision dam deformation predictions.

(3) The MALO-AWLSSVM model has satisfactory performance for accurately identifying the distribution of outliers and achieves high-precision predictions for dam deformation. We can conclude that the proposed model provides a promising prediction strategy that can be applied in the field of dam deformation as well as flood interval prediction, the stock price market, and wind speed forecasting.

\section{Abbreviations}

SVM: $\quad$ Support vector machines

LSSVM: Least squares support vector machines

AWLSSVM: Adaptive weighted least squares support vector machines

MRM: $\quad$ Multiple regression method

RBF: Gaussian radial basis function

ALO: $\quad$ Ant Lion Optimizer

MALO: $\quad$ Modified Ant Lion Optimizer

PSO: $\quad$ Particle swarm optimization

GSA: Gravitational search algorithm

ACO: Ant colony optimization

GWO: Grey wolf optimization

CS: $\quad$ Cuckoo search

GOA: Grasshopper optimization approaches

SSA: $\quad$ Salp swarm algorithm
ME: $\quad$ Maximum error

RMSE: $\quad$ Root mean squared error

MAE: Mean absolute error

MAPE: $\quad$ Mean absolute percentage error

PER: The ratio of the number of outliers to the number of the training sets

AMP: $\quad$ The absolute amplitude of the outlier

PRL: The ratio of the test set to the data set.

\section{Data Availability}

The data used to support the findings of this study have not been made available.

\section{Conflicts of Interest}

The authors declare that they have no conflicts of interest regarding the publication of this paper.

\section{Acknowledgments}

This work was supported by the National Natural Science Foundation of China (Grants nos. 51739003, 51579085, 51779086, 51579086, and 51609074), National Key R\&D Program of China (2018YFC0407104, 2018YFC1508603, 2018YFC0407101, and 2016YFC0401601), Project Funded by the Priority Academic Program Development of Jiangsu Higher Education Institutions (YS11001), Special Project Funded of National Key Laboratory (20165042112), Key R\&D Program of Guangxi (AB17195074), Water Conservancy Science and Technology Project of Jiangsu Province (2019008), and Fundamental Research Funds for the Central Public Welfare Research Institute (Y119002).

\section{References}

[1] J. Jia, "A technical review of hydro-project development in China," Engineering, vol. 2, no. 3, pp. 302-312, 2016.

[2] The Ministry of Water Resources of the People's Republic of China, Bulletin of First National Census for Water, China Water \& Power Press, Beijing, China, 2012.

[3] C. Gu, E. Zhao, and Z. Zhou, Deformation Safety Monitoring of Super High Arch Dams: Theories, Methods and Their Applications, Hohai University Press, Nanjing, China, 2018.

[4] F. Salazar, R. Morán, M. Á. Toledo, and E. Oñate, "Data-based models for the prediction of dam behaviour: a review and some methodological considerations," Archives of Computational Methods in Engineering, vol. 24, no. 1, pp. 1-21, 2017.

[5] J. Mata, "Interpretation of concrete dam behaviour with artificial neural network and multiple linear regression models," Engineering Structures, vol. 33, no. 3, pp. 903-910, 2011.

[6] Z. Wu, Safety Monitoring Theory and Its Application of Hydraulic Structures, Higher Education Press, Beijing, China, 2003.

[7] C. Zhang, T. Peng, J. Zhou, J. Ji, and X. Wang, "An improved autoencoder and partial least squares regression-based extreme learning machine model for pump turbine characteristics," Applied Sciences, vol. 9, no. 19, p. 3987, 2019.

[8] T. Peng, C. Zhang, J. Zhou, X. Xia, and X. Xue, "Multi-objective optimization for flood interval prediction based on orthogonal chaotic NSGA-II and kernel extreme learning 
machine," Water Resources Management, vol. 33, no. 14, pp. 4731-4748, 2019.

[9] X. Liang, Z. Ge, L. Sun, M. He, and H. Chen, "LSTM with wavelet transform based data preprocessing for stock price prediction," Mathematical Problems in Engineering, vol. 2019, Article ID 1340174, 8 pages, 2019.

[10] W. Fu, K. Wang, J. Tan, and K. Zhang, "A composite framework coupling multiple feature selection, compound prediction models and novel hybrid swarm optimizer-based synchronization optimization strategy for multi-step ahead short-term wind speed forecasting," Energy Conversion and Management, vol. 205, Article ID 112461, 2020.

[11] M. Li and J. Wang, "An empirical comparison of multiple linear regression and artificial neural network for concrete dam deformation modelling," Mathematical Problems in Engineering, vol. 2019, Article ID 7620948, 13 pages, 2019.

[12] H. Su, Z. Chen, and Z. Wen, "Performance improvement method of support vector machine-based model monitoring dam safety," Structural Control and Health Monitoring, vol. 23, no. 2, pp. 252-266, 2016.

[13] F. Kang, J. Liu, J. Li, and S. Li, "Concrete dam deformation prediction model for health monitoring based on extreme learning machine," Structural Control and Health Monitoring, vol. 24, no. 10, p. e1997, 2017.

[14] F. Salazar, M. Á. Toledo, E. Oñate, and B. Suárez, "Interpretation of dam deformation and leakage with boosted regression trees," Engineering Structures, vol. 119, pp. 230-251, 2016.

[15] C. Lin, T. Li, S. Chen, X. Liu, C. Lin, and S. Liang, "Gaussian process regression-based forecasting model of dam deformation," Neural Computing and Applications, vol. 31, no. 12, pp. 8503-8518, 2019.

[16] B. Wei, D. Yuan, Z. Xu, and L. Li, "Modified hybrid forecast model considering chaotic residual errors for dam deformation," Structural Control and Health Monitoring, vol. 25, no. 8, p. e2188, 2018.

[17] V. N. Vapnik, The Nature of Statistical Learning Theory, Springer, New York, NY, USA, 1995.

[18] Z. Lu and J. Sun, "Non-Mercer hybrid kernel for linear programming support vector regression in nonlinear systems identification," Applied Soft Computing, vol. 9, no. 1, pp. 94-99, 2009.

[19] J. A. K. Suykens and J. Vandewalle, "Least squares support vector machine classifiers," Neural Processing Letters, vol. 9, no. 3, pp. 293-300, 1999.

[20] J. A. Suykens, J. De Brabanter, L. Lukas, and J. Vandewalle, "Weighted least squares support vector machines: robustness and sparse approximation," Neurocomputing, vol. 48, no. 1-4, pp. 85-105, 2002.

[21] C. Zhang, C. Li, T. Peng et al., "Modeling and synchronous optimization of pump turbine governing system using sparse robust least squares support vector machine and hybrid backtracking search algorithm," Energies, vol. 11, no. 11, p. 3108, 2018.

[22] W. Cui and X. Yan, "Adaptive weighted least square support vector machine regression integrated with outlier detection and its application in QSAR," Chemometrics and Intelligent Laboratory Systems, vol. 98, no. 2, pp. 130-135, 2009.

[23] Y. Zhang, S. Wang, P. Phillips, and G. Ji, "Binary PSO with mutation operator for feature selection using decision tree applied to spam detection," Knowledge-Based Systems, vol. 64, pp. 22-31, 2014.

[24] M. Wang, Y. Wan, Z. Ye, X. Gao, and X. Lai, "A band selection method for airborne hyperspectral image based on chaotic binary coded gravitational search algorithm," Neurocomputing, vol. 273, pp. 57-67, 2018.
[25] S. Kashef and H. Nezamabadi-pour, "An advanced ACO algorithm for feature subset selection," Neurocomputing, vol. 147, pp. 271-279, 2015.

[26] E. Emary, H. M. Zawbaa, and A. E. Hassanien, "Binary grey wolf optimization approaches for feature selection," Neurocomputing, vol. 172, pp. 371-381, 2016.

[27] M. A. E. Aziz and A. E. Hassanien, "Modified cuckoo search algorithm with rough sets for feature selection," Neural Computing and Applications, vol. 29, no. 4, pp. 925-934, 2018.

[28] M. Mafarja, I. Aljarah, A. A. Heidari et al., "Evolutionary population dynamics and grasshopper optimization approaches for feature selection problems," Knowledge-Based Systems, vol. 145, pp. 25-45, 2018.

[29] H. Faris, M. M. Mafarja, A. A. Heidari et al., "An efficient binary salp swarm algorithm with crossover scheme for feature selection problems," Knowledge-Based Systems, vol. 154, pp. 43-67, 2018.

[30] S. Mirjalili, "The ant lion optimizer," Advances in Engineering Software, vol. 83, pp. 80-98, 2015.

[31] E. S. Ali, S. M. Abd Elazim, and A. Y. Abdelaziz, "Ant lion optimization algorithm for optimal location and sizing of renewable distributed generations," Renewable Energy, vol. 101, pp. 1311-1324, 2017.

[32] K. Zhang, J. Ma, X. Zhao, X. Liu, and Y. Zhang, "Parameter identification and state of charge estimation of NMC cells based on improved ant lion optimizer," Mathematical Problems in Engineering, vol. 2019, Article ID 4961045, 18 pages, 2019.

[33] M. M. Mafarja and S. Mirjalili, "Hybrid binary ant lion optimizer with rough set and approximate entropy reducts for feature selection," Soft Computing, vol. 23, no. 15, pp. 6249-6265, 2019.

[34] M. Wang, C. Wu, L. Wang, D. Xiang, and X. Huang, "A feature selection approach for hyperspectral image based on modified ant lion optimizer," Knowledge-Based Systems, vol. 168, pp. 39-48, 2019.

[35] P. Barthelemy, J. Bertolotti, and D. S. Wiersma, "A Lévy flight for light," Nature, vol. 453, no. 7194, pp. 495-498, 2008.

[36] Y. Chen, C. Gu, B. Wu, C. Shao, Z. Wu, and B. Dai, "Inversion modeling of dam-zoning elasticity modulus for heightened concrete dam using ICS-IPSO algorithm," Mathematical Problems in Engineering, vol. 2019, Article ID 9328326, 13 pages, 2019.

[37] N. Cristianini and J. Shawe-Taylor, An Introduction to Support Vector Machines and Other Kernel-Based Learning Methods, Cambridge University Press, Cambridge, UK, 2000.

[38] W. Wang, Z. Xu, W. Lu, and X. Zhang, "Determination of the spread parameter in the Gaussian kernel for classification and regression," Neurocomputing, vol. 55, no. 3-4, pp. 643-663, 2003.

[39] I. Braga, L. P. do Carmo, C. C. Benatti, and M. C. Monard, "A note on parameter selection for support vector machines," in Proceedings of the 2013 Mexican International Conference on Artificial Intelligence, pp. 233-244, Springer, Mexico City, Springer, Berlin, Germany, November 2013.

[40] C. M. Bishop, Pattern Recognition and Machine Learning, Springer, Berlin, Germany, 2006.

[41] X.-S. Yang and S. Deb, "Multiobjective cuckoo search for design optimization," Computers \& Operations Research, vol. 40, no. 6, pp. 1616-1624, 2013.

[42] W. Shiyong, S. Manbin, and W. Jian, "Jinping hydropower project: main technical issues on engineering geology and rock mechanics," Bulletin of Engineering Geology and the Environment, vol. 69, no. 3, pp. 325-332, 2010. 